\title{
DIFFERENTIAL GEOMETRY ON SIMPLICIAL SPACES
}

\author{
BY
}

\author{
MICHAEL A. PENNA
}

\begin{abstract}
A simplicial space $M$ is a separable Hausdorff topological space equipped with an atlas of linearly related charts of varying dimension; for example every polyhedron is a simplicial space in a natural way. Every simplicial space possesses a natural structure complex of sheaves of piecewise smooth differential forms, and the homology of the corresponding de Rham complex of global sections is isomorphic to the real cohomology of $M$.

A cosimplicial bundle is a continuous surjection $\xi: E \rightarrow M$ from a topological space $E$ to a simplicial space $M$ which satisfies certain criteria. There is a category of cosimplicial bundles which contains a subcategory of vector bundles. To every simplicial space $M$ a cosimplicial bundle $\tau(M)$ over $M$ is associated; $\tau(M)$ is the cotangent object of $M$ since there is an isomorphism between the module of global piecewise smooth one-forms on $M$ and sections of $\tau(M)$.
\end{abstract}

Introduction. The goal of this paper is to provide some foundations for the study of differential geometry on polyhedra.

Simplicial spaces and simplicial maps are defined in $\S 1$. These spaces are generalized polyhedra and the main objects of study in this paper. In $\S 3$ the structure algebra $A(M)$ of piecewise smooth functions on the simplicial space $M$ is developed in two ways. The first approach is direct: $A(M)$ is the algebra $\Gamma(M, \mathbf{A}(M))$ of global sections of the sheaf $\mathbf{A}(M)$ associated to the presheaf which assigns to each open subset $U$ of $M$ the algebra $A(U)$ of continuous piecewise smooth real valued functions defined on $U$. The second approach is indirect: $U$ is viewed as being patched together and $A(U)$ is viewed as an algebra of compatible tuples of piecewise smooth functions defined on the components of $U$. This second approach motivates the development of the $A(M)$-modules $\Lambda^{q} E(M)$ of piecewise smooth $q$ forms on $M, q=1,2, \ldots: \Lambda^{q} E(M)$ is the module $\Gamma\left(M, \Lambda^{q} \mathrm{E}(M)\right)$ of global sections of the sheaf $\Lambda^{q} \mathrm{E}(M)$ associated to the presheaf which assigns to each open subset $U$ of $M$ a module $\Lambda^{q} E(U)$ of compatible tuples of piecewise smooth $q$ forms defined on the components of $U$. There is a derivation defined on these forms which generates a de Rham complex $\left(\Lambda^{*} E(M), d(M)\right)$. The de Rham theorem for simplicial spaces states that the homology of this cochain complex is

Received by the editors January 29, 1974 and, in revised form, October 9, 1974 and February 11, 1975.

AMS (MOS) subject classifications (1970). Primary 18F10, 57B99, 58A10, 55F10.

Key words and phrases. Polyhedron, manifold, differential forms, de Rham theorem, cotangent bundle. 
isomorphic to the real cohomology of $M$.

A cosimplicial bundle, defined in $\S 5$, is a continuous surjection $\xi: E \rightarrow M$ from a topological space $E$ to a simplicial space $M$ which satisfies certain criteria. There is a category of cosimplicial bundles which contains a subcategory of vector bundles. In general, however, cosimplicial bundles differ from vector bundles since fiber dimensions in cosimplicial bundles are allowed to vary. A section $s$ of a cosimplicial bundle $\xi: E \rightarrow M$ is a (not necessarily continuous) function $s: M \rightarrow E$ satisfying certain criteria; the set $\Gamma(M, \xi)$ of global sections of $\xi$ forms an $A(M)$ module. In the same way every smooth manifold has a related cotangent object in the category of smooth vector bundles, every simplicial space $M$ has a related cotangent object $\tau(M)$ in the category of cosimplicial bundles. The cosimplicial bundle $\tau(M)$ is a cotangent object since there is an $A(M)$-module isomorphism between the module $E(M)$ of global piecewise smooth one-forms on $M$ and the module $\Gamma(M, \tau(M))$ of global sections of $\tau(M)$. Such cotangent objects are not usually vector bundles.

An application of this approach to the study of polyhedra is outlined in $\S 6$. Other applications will appear elsewhere.

1. Simplicial spaces. In the classical sense manifolds are topological spaces $M$ covered by open sets $U$ with homeomorphisms $\psi: U \rightarrow V$ onto open subsets $V$ of a fixed Euclidean space $R^{n}$ where $R$ is the real field and each pair $(U, \psi)$ is a chart in an atlas of $M$. By requiring the homeomorphisms

$$
\left(\left.\psi_{2}\right|_{U_{1} \cap U_{2}}\right) \circ\left(\left.\psi_{1}\right|_{U_{1} \cap U_{2}}\right)^{-1}: \psi_{1}\left(U_{1} \cap U_{2}\right) \rightarrow \psi_{2}\left(U_{1} \cap U_{2}\right)
$$

of open subsets of $R^{n}$ to be smooth or PL one then specifies that $M$ is smooth or PL, for example. In what follows we consider spaces $M$ which are not manifolds and charts $(U, \psi)$ in which the domains $U \subseteq M$ of the homeomorphisms $\psi$ are not necessarily open subsets of $M$ in a similar manner.

Let $e_{1}, \ldots, e_{n}$ be the standard basis vectors of $R^{n}$ and let $x_{1}, \ldots, x_{n}$ be the standard coordinate functions on $R^{n}$.

1.1. Definition. For any $p=1, \ldots, n$ and any distinct $e_{i_{1}}, \ldots, e_{i_{p}}$, the $p$-comer $s_{i}$ spanned by $e_{i_{1}}, \ldots, e_{i_{p}}$ is the set

$$
s_{i}=\left\{x=\sum x_{k} e_{k} \in R^{n}: x_{k} \in R^{+} \text {for } k \in i \text { and } x_{k}=0 \text { otherwise }\right\}
$$

where $R^{+}$is the set of nonnegative real numbers and $i$ denotes the $p$-tuple $\left(i_{1}\right.$, $\left.\ldots, i_{p}\right)$. For convenience we write $s^{n}$ for $s_{(1, \ldots, n)}$. The 0 -corner $s^{0}$ is the point $R^{0}$ itself.

Thus in the symbol $s^{i}$ the superscript $i$ is a natural number while in the symbol $s_{i}$ the subscript $i$ is a $p$-tuple.

In what follows we use the term "linear transformation" by abuse of language 
to indicate restrictions of affine transformations (both domains and ranges being restricted).

1.2. Definition. Let $M$ be a topological space. A simplicial chart on $M$ is a pair $(U, \psi)$ consisting of a nonempty subset $U$ of $M$ and a homeomorphism $\psi: U \rightarrow V$ where $V$ is an open subset of some $i$-corner $s$. Two simplicial charts $\psi_{\alpha}: U_{\alpha} \rightarrow V_{\alpha} \subseteq s_{\alpha}^{i}$ and $\psi_{\beta}: U_{\beta} \rightarrow V_{\beta} \subseteq s_{\beta}^{j}$ are related iff $\psi_{\alpha}\left(U_{\alpha} \cap U_{\beta}\right)$ and $\psi_{\beta}\left(U_{\alpha} \cap U_{\beta}\right)$ are open subsets of $k$-faces $s_{\alpha \beta}^{i}$ and $s_{\beta \alpha}^{j}$ of $s_{\alpha}^{i}$ and $s_{\beta}^{j}$, respectively, for some $k$ which depends on $U_{\alpha}$ and $U_{\beta}$, and for which

$$
\left(\psi_{\beta} \mid U_{\alpha} \cap U_{\beta}\right) \circ\left(\psi_{\alpha} \mid U_{\alpha} \cap U_{\beta}\right)^{-1}: \psi_{\alpha}\left(U_{\alpha} \cap U_{\beta}\right) \rightarrow \psi_{\beta}\left(U_{\alpha} \cap U_{\beta}\right)
$$

is an invertible linear transformation. A simplicial atlas on $M$ is a collection of related simplicial charts on $M$ whose domains cover $M$ and which is maximal with respect to the inclusion of related simplicial charts. An $n$-dimensional simplicial space $M$ is a separable Hausdorff topological space equipped with a simplicial atlas such that

(1) if $\psi: U \rightarrow V$ is a simplicial chart in the atlas then $\operatorname{dim} V \leqslant n$, and

(2) each point in $M$ has an open neighborhood which may be written as a finite union of domains of simplicial charts in the atlas.

1.3. REMARK. In the definition of "related" the subsets $U_{\alpha}$ and $U_{\beta}$ are not required to have the same dimension in any sense and the notation $s_{\alpha}^{i}$ emphasizes this. We will suppress the superscript $i$, however, and write $s_{\alpha}$ for $s_{\alpha}^{i}$ since the dimension $i$ is given implicitly in the description of a simplicial chart.

1.4. Definition. Let $M_{1}$ and $M_{2}$ be simplicial spaces. A simplicial map $f: M_{1} \rightarrow M_{2}$ of simplicial spaces is a continuous function such that

(1) for every simplicial chart $\left(U_{1}, \psi_{1}\right)$ on $M_{1}$ there is a simplicial chart $\left(U_{2}, \psi_{2}\right)$ on $M_{2}$ for which $f\left(U_{1}\right) \subseteq U_{2}$, and

(2) for all simplicial charts $\left(U_{1}, \psi_{1}\right)$ on $M_{1}$ and $\left(U_{2}, \psi_{2}\right)$ on $M_{2}$, the map $\left(\psi_{2} \mid f\left(U_{1}\right) \cap U_{2}\right) \circ\left(f U_{U_{1}}\right) \circ\left(\psi_{1}\right)^{-1}$ is a linear transformation.

Simplicial spaces and simplicial maps form a category.

1.5. Proposition. There is a natural inclusion of the category of locally finite, finite dimensional simplicial complexes and simplicial maps (or the category of polyhedra for short) as a subcategory of the category of simplicial spaces.

Proof. Let $K$ be a simplicial complex and $|K|$ its geometric realization. For every $p$-simplex $\tau$ of $K$ there are linear homeomorphisms $h:|\tau| \rightarrow \Delta^{p}$ from the geometric realization $|\tau|$ of $\tau$ to the standard $p$-simplex

$$
\Delta^{p}=\left\{x \in R^{p}: \sum x_{j} \leqslant 1 \text { and } x_{j} \geqslant 0 \forall j\right\} \subseteq R^{p} .
$$

For $x_{0} \in|K|$ let St $x_{0}$ be the open star of $x_{0}$ in $|K|$. Then $|K|$ is a simplicial 
space with respect to the atlas generated by all simplicial charts of the form $\left(|\tau| \cap\right.$ St $\left.x_{0},\left.h\right|_{|\tau| \cap S t x_{0}}\right)$. Q.E.D.

There are simplicial spaces which cannot be considered as polyhedra. For example consider any $n$-corner $s^{n}, n>0$, as a simplicial space whose atlas is generated by the single simplicial chart id: $s^{n} \rightarrow s^{n}$ (the identity). If $s^{n}$ could be considered as a polyhedron then there would have to be points of $s^{n}$ corresponding to vertices other than the origin. If $x_{0}$ were such a point then $(U, \psi)$ would be a simplicial chart on $s^{n}$ where $U=\left\{x_{0}\right\}$ and $\psi: U \rightarrow s^{0}$. But this is impossible since if $\left(s^{n}\right.$, id) and $(U, \psi)$ were related simplicial charts, the point $x_{0} \in s^{n}$ would have to be a 0 -face of $s^{n}$; however the origin is the only 0 -face of $s^{n}$ and $x_{0}$ was chosen to be other than the origin. (Other examples of simplicial spaces which cannot be considered as polyhedra are CW-complexes or $R^{n}$ for any $n>0$.)

Loosely speaking then, a simplicial space is simply a topological space equipped with a specific simplicial structure. It seems reasonable to conjecture that every simplicial space $M$ has a subdivision with respect to which $M$ is a polyhedron. As we will see, however, there are important geometric differences between a simplicial space $M$ and a subdivision of $M$. In this sense simplicial spaces are super polyhedra.

Let $x_{0}$ be a point of the simplicial space $M$. Then for every simplicial chart $\psi_{\alpha}: U_{\alpha} \rightarrow V_{\alpha} \subseteq s_{\alpha}$ on $M, U_{\alpha}$ containing $x_{0}, \psi_{\alpha}\left(x_{0}\right)$ is contained in the interior of a unique face $s_{\alpha}\left(x_{0}\right)$ of $s_{\alpha}$.

1.6. Definition. An open neighborhood $U$ of $x_{0}$ is small iff $U$ has compact closure and $U$ may also be written as the (finite) union of domains $U_{\alpha}$ of simplicial charts $\psi_{\alpha}: U_{\alpha} \rightarrow V_{\alpha} \subseteq s_{\alpha}$ on $M, U_{\alpha}$ containing $x_{0}$, such that each $V_{\alpha}$ meets no face of $s_{\alpha}$ of dimension less than or equal to that of $s_{\alpha}\left(x_{0}\right)$, except $s_{\alpha}\left(x_{0}\right)$ itself of course.

For example (see Diagram 1) if $M$ is a polyhedron then a small open neighborhood $U$ of $x_{0} \in M$ is the intersection of any open neighborhood of $x_{0}$ with the open star St $x_{0}$ of $x_{0}$ in $M$.

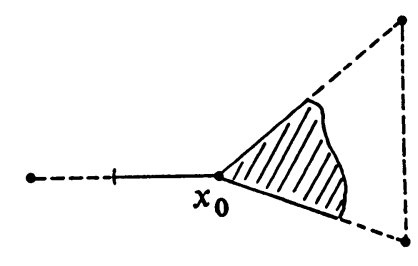

\section{DiAgRAM 1}

1.7. Definition. A small open subset $U$ of the simplicial space $M$ is an open subset of $M$ which is a small open neighborhood of some $x_{0} \in U$.

1.8. REMARK. The set of small open neighborhoods of the point $x_{0} \in M$ 
forms a complete neighborhood system at $x_{0}$ for the topology of $M$, and the set of small open subsets of $M$ forms a basis for the topology of $M$.

The definition of simplicial space differs from the definitions of smooth and PL manifolds, for example, since the domains of charts on a simplicial space are not necessarily open. The following redefinition of simplicial spaces by "packets" remedies this, and is also required for later purposes.

1.9. Definition. Let $M$ be a topological space. A packet on $M$ is a pair $(U, \Psi)$ consisting of a nonempty open subset $U$ of $M$ which has compact closure and a collection $\Psi$ of related simplicial charts $\left(U_{\alpha}, \psi_{\alpha}\right)$ on $M, \alpha$ in some index set $A$, where $U=\bigcup U_{\alpha}$, the union taken over all $\alpha$ in $A$, and for which

(1) $U_{\alpha}$ is an open subset of $U_{\beta}$ iff $\alpha=\beta$, and

(2) (maximality) for each $\alpha, \beta \in A$ there is a $\gamma \in A$ for which $U_{\alpha} \cap U_{\beta}$ $=U_{\gamma}$.

The prototype of a packet is the open star St $x_{0}$ of a point $x_{0} \in M, M$ a polyhedron, equipped with the decomposition into subsets determined by the simplices of $M$. Diagram 2 illustrates the natural packet structure on the small open subset of Diagram 1.

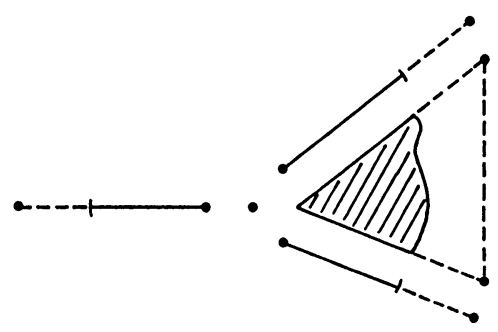

Diagram 2

1.10. Definition. Two packets $\left(U^{\prime}, \Psi^{\prime}\right)$ and $\left(U^{\prime \prime}, \Psi^{\prime \prime}\right)$ are related whenever each simplicial chart of $\Psi^{\prime}$ is related to each simplicial chart of $\Psi^{\prime \prime}$. A (packet) simplicial atlas on the topological space $M$ is a collection of related packets on $M$ whose "domains" cover $M$ and which is maximal with respect to the inclusion of related packets. An n-dimensional (packet) simplicial space $M$ is a separable Hausdorff topological space equipped with a (packet) simplicial atlas such that

(1) if $\psi_{\alpha}: U_{\alpha} \rightarrow V_{\alpha}$ is any simplicial chart of any packet $(U, \Psi)$ in the atlas then $\operatorname{dim} V_{\alpha} \leqslant n$, and

(2) the index set $A$ of each packet $(U, \Psi)$ in the atlas is finite.

We write (packet) simplicial space to distinguish from (simplicial chart) simplicial space. We soon show that these two definitions are equivalent and thereafter drop any mention of the particular definition used.

1.11. REMARK. If $(U, \Psi)$ is a packet on the (packet) simplicial space $M$ then $U$ is a small open subset of $M$ : If not then there are two simplicial charts 
$\psi_{\alpha}: U_{\alpha} \rightarrow V_{\alpha}$ and $\psi_{\beta}: U_{\beta} \rightarrow V_{\beta}$ of $\Psi$ such that $n=\operatorname{dim} V_{\alpha}=\operatorname{dim} V_{\beta}$ and such that for every other simplicial chart $\psi_{\gamma}: U_{\gamma} \rightarrow V_{\gamma}$ of $\Psi, n \leqslant \operatorname{dim} V_{\gamma}$. By "maximality", $U_{\alpha} \cap U_{\beta}=\varnothing$ would have to be the domain of some simplicial chart of $\Psi$; but this is impossible since the domains of simplicial charts are required to be nonempty. Thus every packet $(U, \Psi)$ contains a simplicial chart $\left(U_{0}, \psi_{0}\right)$ for which $U_{0} \subseteq U_{\alpha}$ for every other simplicial chart $\left(U_{\alpha}, \psi_{\alpha}\right)$ of $\Psi$; in fact $U_{0}$ is precisely the set of points with respect to which $U$ is a small open neighborhood.

1.12. REMARK. If $\left(U, \Psi^{\prime}\right)$ and $\left(U, \Psi^{\prime \prime}\right)$ are related packets on the same small open subset $U$ then the domains of the simplicial charts of $\Psi^{\prime}$ and $\Psi^{\prime \prime}$ are the same: If $\left(U_{\alpha}, \psi_{\alpha}\right)$ is a simplicial chart of $\Psi^{\prime}$ then $U_{\alpha}=\bigcup\left(U_{\alpha} \cap U_{\beta}\right)$ where the $U_{\beta}$ are the domains of the simplicial charts $\left(U_{\beta}, \psi_{\beta}\right)$ of $\Psi^{\prime \prime}$. Since $\left(U_{\alpha}, \psi_{\alpha}\right)$ is related to each simplicial chart $\left(U_{\beta}, \psi_{\beta}\right)$ of $\Psi^{\prime \prime}$, each $U_{\alpha} \cap U_{\beta}$ is the domain of some simplicial chart of $\Psi^{\prime}$. Thus there must be a simplicial chart $\left(U_{\beta}, \psi_{\beta}\right)$ of $\Psi^{\prime \prime}$ for which $U_{\alpha}=U_{\alpha} \cap U_{\beta}$; that is, $U_{\alpha} \subseteq U_{\beta}$. Since $\left(U_{\alpha}, \psi_{\alpha}\right)$ and $\left(U_{\beta}, \psi_{\beta}\right)$ are related simplicial charts and since packets in general (and $\Psi^{\prime \prime}$ in particular) are "maximal", $U_{\alpha}$ is the domain of some simplicial chart of $\Psi$ ".

\subsection{Proposition. The definition of (simplicial chart) simplicial space} is equivalent to the definition of (packet) simplicial space.

Proof. If $M$ is defined by packets then by ignoring the structure of the packets as collections of related simplicial charts we may consider $M$ to be described by simplicial charts.

Next assume that $M$ is defined by simplicial charts. Let $U$ be a small open subset of $M$ and $x_{0} \in U$ a point with respect to which $U$ is a small open neighborhood. By Definition 1.6, $U$ can be written as the union of sets of the form $U_{i}, i$ in some finite index set $I$, where $\psi_{i}: U_{i} \rightarrow V_{i} \subseteq s_{i}$ are simplicial charts on $M$ such that $x_{0}$ is contained in each $U_{i}$ and such that each $V_{i}$ meets no face of $s_{i}$ of dimension less than or equal to that of $s_{i}\left(x_{0}\right)$, except $s_{i}\left(x_{0}\right)$ itself, of course. If $\left(U_{i}, \psi_{i}\right)$ and $\left(U_{j}, \psi_{j}\right)$ are simplicial charts in $\left\{\left(U_{i}, \psi_{i}\right)\right\}_{i \in I}$ for which $\psi_{i}\left(U_{i} \cap U_{j}\right)$ and $\psi_{j}\left(U_{i} \cap U_{j}\right)$ are nonempty open subsets of $\psi_{i}\left(U_{i}\right)$ and $\psi_{j}\left(U_{j}\right)$, respectively, then since $U$ is compact and the map $\left(\psi_{j} \mid U_{i} \cup U_{j}\right) \circ\left(\left.\psi_{i}\right|_{U_{i} \cap U_{j}}\right)^{-1}$ from $\psi_{i}\left(U_{i} \cap U_{j}\right)$ to $\psi_{j}\left(U_{i} \cap U_{j}\right)$ is an invertible linear transformation, there is a simplicial chart of the form $\left(U_{i} \cup U_{j}, \psi\right)$ on $M$. Consequently the set $I$ may be partitioned into subsets $I_{\alpha}$ in such a way that $i$ and $j$ are both in $I_{\alpha}$ iff there is a simplicial chart of the form $\left(U_{i} \cup U_{j}, \psi\right)$ on $M$ for which $\psi\left(U_{i}\right)$ and $\psi\left(U_{j}\right)$ are both open subsets of $\psi\left(U_{i} \cup U_{j}\right)$. We can associate a simplicial chart $\left(U_{\alpha}, \psi_{\alpha}\right)$ to each set $U_{\alpha}=$ $\bigcup U_{i}$, the union taken over all $i \in I_{\alpha}$, and "maximizing" the resulting set $\left\{\left(U_{\alpha}\right.\right.$, $\left.\psi_{\alpha}\right)$ \} of simplicial charts we obtain a packet structure on $U$.

Having done this for each small open subset $U$ of $M$ we obtain a collection 
of packets on $M$ whose "domains" cover $M$ and which are related a priori. By adding to this collection all related packets we may describe $M$ by means of a (packet) simplicial atlas and thus consider $M$ as a (packet) simplicial space.

These two processes are trivially inverses of each other. Q.E.D.

We henceforth drop any specific mention of simplicial charts or packets when speaking of simplicial spaces.

1.14. Remark. As a result of Remark 1.11 and the proof of Proposition 1.13 , a subset $U$ of the simplicial space $M$ is the "domain" of some packet $(U, \Psi)$ on $M$ iff $U$ is a small open subset of $M$.

1.15. RemarK. If $(U, \Psi)$ is a packet on the simplicial space $M$ and $W$ is a small open subset of $U$ then $\Psi$ induces a packet structure on $W$ which is described by the set of all simplicial charts of the form $\left(W \cap U_{\alpha}, \psi_{\alpha} \mid w \cap U_{\alpha}\right)$ for $\left(U_{\alpha}, \psi_{\alpha}\right)$ a simplicial chart of $\Psi$ for which $W \cap U_{\alpha}$ is nonempty.

2. Piecewise smooth forms. The purpose of this section is to introduce the real analysis necessary for the development of the de Rham complexes on simplicial spaces.

Let $D$ be the real linear space spanned by differential operators

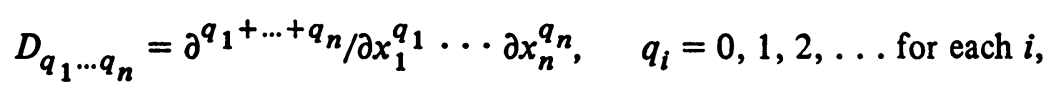

defined on the set of smooth real valued functions defined on $R^{n}$.

2.1. Definition. The smooth real valued function $f:$ Int $s^{n} \rightarrow R$ defined on the interior Int $s^{n}$ of the $n$-corner $s^{n}$ is closure smooth iff for every $x_{0} \in s^{n}$, the limit of $D f(x)$ at $x_{0}$ is finite for every $D \in D$. A closure smooth $q$-form defined on Int $s^{n}$ is any smooth $q$-form $\Sigma f_{i} d x_{i_{1}} \cdots d x_{i_{q}}$ defined on Int $s^{n}$ for which each $f_{i}$ is a closure smooth function on Int $s^{n}$.

The closure smooth functions defined on Int $s^{n}$ form a subalgebra of the algebra of smooth functions defined on Int $s^{n}$. There is also a natural injection of the module of closure smooth $q$-forms defined on Int $s^{n}$ (over the algebra of closure smooth functions defined on Int $s^{n}$ ) into the module of smooth $q$-forms defined on Int $s^{n}$ (over the algebra of smooth functions defined on Int $s^{n}$ ). The standard derivation of smooth forms defined on Int $s^{n}$ induces a derivation of closure smooth forms defined on Int $s^{n}$ for which there is a natural injection of the closure smooth forms defined on Int $s^{n}$ as a cochain subcomplex of the cochain complex of smooth forms defined on Int $s^{n}$.

2.2. Definition. The algebra $A\left(s^{n}\right)$ of piecewise smooth functions defined on the n-corner $s^{n}$ is the algebra of continuous real valued functions $f: s^{n}$ $\rightarrow R$ such that $f$ restricted to the interior Int $s_{i}$ of every face $s_{i}$ of $s^{n}$ is closure smooth. 
2.3. LEMMA. Every closure smooth function $f:$ Int $s^{n} \rightarrow R$ has a unique extension to a piecewise smooth function $f: s^{n} \rightarrow R$.

Proof. Define $f$ at the point $x_{0}$ on the boundary of $s^{n}$ as the limit of $f(x)$ at $x_{0}$. The remainder of the lemma is immediate. Q.E.D.

2.4. Definition. The module $E\left(s^{n}\right)$ of piccewise smooth 1-forms on the $n$-corner $s^{n}$ is the free $A\left(s^{n}\right)$-module on generators $d x_{1}, \ldots, d x_{n}$. The derivation $d\left(s^{n}\right): A\left(s^{n}\right) \rightarrow E\left(s^{n}\right)$ is given as follows: Each $f \in A\left(s^{n}\right)$ is smooth on Int $s^{n}$. For $i=1, \ldots, n, \partial f / \partial x_{i}$ is defined on Int $s^{n}$ and, by Lemma 2.3 , it may be extended to a unique piecewise smooth function, again denoted by $\partial f / \partial x_{i}$, defined on all of $s^{n}$. Let $d\left(s^{n}\right)(f)=\Sigma\left(\partial f / \partial x_{i}\right) d x_{i}$.

2.5. Definition. For $q=1,2, \ldots$, the $A\left(s^{n}\right)$-module $\Lambda^{q} E\left(s^{n}\right)$ of piecewise smooth $q$-forms on the $n$-corner $s^{n}$ is the $q$-fold exterior product of $E\left(s^{n}\right)$, and the derivation $d\left(s^{n}\right): \Lambda^{q} E\left(s^{n}\right) \rightarrow \Lambda^{q+1} E\left(s^{n}\right)$ is given by

$$
d\left(s^{n}\right)\left(\sum_{i} f_{i} d x_{i_{1}} \cdots d x_{i_{q}}\right)=\sum_{i, i_{0}}\left(\partial f_{i} / \partial x_{i_{0}}\right) d x_{i_{0}} d x_{i_{1}} \cdots d x_{i_{q}} .
$$

For convenience we frequently write the $q$-form

$$
\sum f_{i} d x_{i_{1}} \cdots d x_{i_{q}} \in \Lambda^{q} E\left(s^{n}\right)
$$

as $\Sigma f_{i} d x_{i}$ where $i=\left(i_{1}, \ldots, i_{q}\right)$ and $d x_{i}=d x_{i_{1}} \cdots d x_{i_{q}}$.

Since restrictions of piecewise smooth forms defined on $s^{n}$ to closure smooth forms defined on Int $s^{n}$ and extension of closure smooth forms defined on Int $s^{n}$ to piecewise smooth forms defined on $s^{n}$ are inverse processes, and since the derivation $d\left(s^{n}\right)$ is a natural extension of the standard derivation of closure smooth forms defined on Int $s^{n}, d\left(s^{n}\right) \circ d\left(s^{n}\right)=0$. Consequently $\left(\Lambda^{*} E\left(s^{n}\right), d\left(s^{n}\right)\right)$ is a cochain complex.

The preceding concepts can be relativized for any face $s_{i}$ of $s^{n}$ to obtain the cochain complex $\left(\Lambda^{*} E\left(s_{i}\right), d\left(s_{i}\right)\right)$ of piecewise smooth forms defined on $s_{i}$. Notice that the restriction $f_{s_{i}}: s_{i} \rightarrow R$ of a piecewise smooth function $f: s^{n} \rightarrow R$ to the face $s_{i}$ of $s^{n}$ is clearly a piecewise smooth function.

2.6. Definition. The homomorphism $\rho\left(s_{i}, s^{n}\right): A\left(s^{n}\right) \rightarrow A\left(s_{i}\right)$ given by $\rho\left(s_{i}, s^{n}\right)(f)=f_{\mid s_{i}}$ is the map induced by restriction. For each $q=1,2, \ldots$, the module homomorphism $\rho^{q}\left(s_{i}, s^{n}\right): \Lambda^{q} E\left(s^{n}\right) \rightarrow \Lambda^{q} E\left(s_{i}\right)$ given by

$$
\begin{aligned}
\rho^{q}\left(s_{i}, s^{n}\right) & \left(\sum f_{i} d x_{i_{1}} \cdots d x_{i_{q}}\right) \\
= & \sum\left(\rho\left(s_{i}, s^{n}\right) f_{i}\right) d\left(\rho\left(s_{i}, s^{n}\right) x_{i_{1}}\right) \cdots d\left(\rho\left(s_{i}, s^{n}\right) x_{i_{q}}\right)
\end{aligned}
$$

is the map of $q$-forms induced by restriction. 
In general if $s_{i}$ is a face of $s_{j}$, restriction gives a homomorphism $\rho\left(s_{i}, s_{j}\right)$ : $A\left(s_{j}\right) \rightarrow A\left(s_{i}\right)$ and for each $q=1,2, \ldots$ a module homomorphism $\rho^{q}\left(s_{i}, s_{j}\right)$ : $\Lambda^{q} E\left(s_{j}\right) \rightarrow \Lambda^{q} E\left(s_{i}\right)$.

2.7. Proposition. If $s_{i}$ is a face of $s_{j}$ then restriction induces a homomorphism

$$
\rho^{*}\left(s_{i}, s_{j}\right):\left(\Lambda^{*} E\left(s_{j}\right), d\left(s_{j}\right)\right) \rightarrow\left(\Lambda^{*} E\left(s_{i}\right), d\left(s_{i}\right)\right)
$$

of cochain complexes, and if $s_{j}$ is furthermore a face of $s_{k}$ (so that $s_{i}$ is a face of $s_{k}$ ) then there is a commutative diagram of cochain complexes: $\rho^{*}\left(s_{i}, s_{k}\right)=$ $\rho^{*}\left(s_{i}, s_{j}\right) \circ \rho *\left(s_{j}, s_{k}\right)$.

Proof. It suffices to consider the case of the face $s_{i}$ of $s^{n}$. Let $\theta=$ $\Sigma f_{j} d x_{j} \in \Lambda^{q} E\left(s^{n}\right)$. Decompose $\theta$ into components parallel to $s_{i}$ and normal to $s_{i}: \theta=P \theta+N \theta$ where $P \theta=\Sigma^{\prime} f_{j} d x_{j}$, the summation taken over all $j \subseteq i$, and $N \theta=\theta-P \theta$. Notice that $\rho^{q}\left(s_{i}, s^{n}\right)(\theta)=(P \theta)_{\mid s i}$. The derivation $d\left(s^{n}\right): \Lambda^{q} E\left(s^{n}\right)$ $\rightarrow \Lambda^{q+1} E\left(s^{n}\right)$ can be decomposed into components parallel to $s_{i}$ and normal to $s_{i}: d\left(s^{n}\right)=d_{P}\left(s^{n}\right)+d_{N}\left(s^{n}\right)$ where $d_{P}\left(s^{n}\right)\left(f d x_{j}\right)=\Sigma\left(\partial f / \partial x_{j_{0}}\right) d x_{j_{0}} d x_{j}$, the summation taken over all $j_{0} \in i$, and $d_{N}\left(s^{n}\right)=d\left(s^{n}\right)-d_{P}\left(s^{n}\right)$. Finally there is a commutative diagram: $\rho^{q+1}\left(s_{i}, s^{n}\right) \circ d\left(s^{n}\right)=d\left(s_{i}\right) \circ \rho^{q}\left(s_{i}, s^{n}\right)$. For if $\theta \in \Lambda^{q} E\left(s^{n}\right)$ then $d\left(s^{n}\right)(\theta)=d_{P}\left(s^{n}\right)(P \theta)+d_{P}\left(s^{n}\right)(N \theta)+d_{N}\left(s^{n}\right)(P \theta)+d_{N}\left(s^{n}\right)(N \theta)$. Since $P d\left(s^{n}\right)(\theta)=d_{P}\left(s^{n}\right)(P \theta), \rho^{q+1}\left(s_{i}, s^{n}\right)\left(d\left(s^{n}\right)(\theta)\right)=\left.\left(d_{P}\left(s^{n}\right)(P \theta)\right)\right|_{s_{i}}$. However $\rho^{q}\left(s_{i}, s^{n}\right)(\theta)=\left.(P \theta)\right|_{s_{i}}$ and $d\left(s_{i}\right)\left(\left.(P \theta)\right|_{s_{i}}\right)=\left.\left(d_{P}\left(s^{n}\right)(P \theta)\right)\right|_{s_{i}}$ since for all $j_{0} \in i$, $\left.\left(\partial f / \partial x_{j_{0}}\right)\right|_{s_{i}}=\partial\left(f_{s_{i}}\right) / \partial x_{j_{0}}$. Q.E.D.

The preceding concepts can be relativized to arbitrary open subsets $U \subseteq s^{n}$ in the obvious way.

2.8. Lemma (PoINCARÉ). Let $U$ be a small star shaped open neighborhood of the point $x_{0} \in s^{n}$. If $\theta \in \Lambda^{q} E(U)$ is such that $d\left(s^{n}\right)(\theta)=0$ then there is a $\phi \in \Lambda^{q-1} E(U)$ for which $d\left(s^{n}\right)(\phi)=\theta$.

Proof. Proceed along essentially the same lines as in the classical proof (see [1]). Indeed if $r:[0,1] \times U \rightarrow U$ is the retraction given by $r(t, x)=$ $x_{0}+t\left(x-x_{0}\right)$ and $K: \Lambda^{q+1} E([0,1] \times U) \rightarrow \Lambda^{q} E(U)$ is given by $K\left(f(t, x) d x_{k}\right)$ $=0$ and $K\left(f(t, x) d t d x_{k}\right)=\left(\int_{t=0}^{t=1} f(t, x) d t\right) d x_{k}$ then $\phi=K r^{*} \theta$. The point is that since $r$ is linear, $r(t, x)$ is in the face $s_{i}$ of $s^{n}$ for any $t \in[0,1]$ if and only if $x$ is in $s_{i}$. Q.E.D.

2.9. REMARK. With the notation of Lemma 2.8, if $T: U \rightarrow s^{n}$ is an invertible simplicial map taking the faces of $U$ piecewise linearly to the faces of $s^{n}$ then for $\phi=K r^{*} \theta \in \Lambda^{q-1} E(U), d\left(s^{n}\right)(\phi)=\theta$. But $T^{*} \theta \in \Lambda^{q} E\left(T^{-1}(U)\right)$ and $d\left(s^{n}\right)\left(T^{*} \theta\right)=0$ so that if $\phi^{\prime}=K r^{*}\left(T^{*} \theta\right) \in \Lambda^{q-1} E\left(T^{-1}(U)\right)$ then $d\left(s^{n}\right)\left(\phi^{\prime}\right)=T^{*} \theta$ (linear transformations preserve the star shaped property). But since $T$ is linear 
and $K$ is independent of $T^{*}, \phi^{\prime}=K r^{*} T^{*} \theta=K T^{*} r^{*} \theta=T^{*} K r^{*} \theta$ so that $\phi^{\prime}=T^{*} \phi$.

2.10. REMARK. With the notation of Lemma 2.8 , if $s_{i}$ is a face of $s^{n}$ containing $x_{0}$ then $U \cap s_{i}$ is a small open neighborhood of $x_{0}$ in $s_{i}$ and $d\left(s_{i}\right)\left(\rho^{q}\left(s_{i}, s^{n}\right)(\theta)\right)=0$. If $\phi^{\prime}=K r^{*}\left(\rho^{q}\left(s_{i}, s^{n}\right) \theta\right)$ then $d\left(s_{i}\right)\left(\phi^{\prime}\right)=\rho^{q}\left(s_{i}, s^{n}\right) \theta$. But moreover $\phi^{\prime}=\rho^{q-1}\left(s_{i}, s^{n}\right) \phi$ : If $P$ superscripts denote parallel coordinate functions and $N$ superscripts denote normal coordinate functions then $P\left(K r^{*}(N \theta)\right)$ has summands of the form

$$
x_{j_{1}}^{N}\left(\int_{t=0}^{t=1} f_{j}(t \cdot x) d t\right) d x_{j_{2}}^{P} \cdots d x_{j_{q}}^{P}
$$

and since $\left.x_{j_{1}}^{N}\right|_{s_{i}}=0,\left.P\left(K r^{*}(N \theta)\right)\right|_{s_{i}}=0$. Thus

$$
\begin{aligned}
\rho^{q-1}\left(s_{i}, s^{n}\right)(\theta) & =\left.\left(P\left(K r^{*} \theta\right)\right)\right|_{s_{i}}=\left.\left(P\left(K r^{*}(P \theta+N \theta)\right)\right)\right|_{s_{i}} \\
& =\left.\left(P\left(K r^{*}(P \theta)\right)\right)\right|_{s_{i}}+\left.\left(P\left(K r^{*}(N \theta)\right)\right)\right|_{s_{i}} \\
& =\left.\left(K r^{*}(P \theta)\right)\right|_{s_{i}}=K r^{*}\left(\left.(P \theta)\right|_{s_{i}}\right)=\phi^{\prime} .
\end{aligned}
$$

3. The de Rham theorem. Let $M$ be a simplicial space.

3.1. REMARK. Since the set of small open subsets of a simplicial space $M$ is a basis for the topology of $M$, a presheaf on $M$ may be described as a contravariant functor defined on the category of small open subsets of $M$ (whose morphisms are inclusions).

3.2. Definition. A continuous function $f: U \rightarrow R$ defined on the small open subset $U$ of the simplicial space $M$ is piecewise smooth (simplicial) iff for every simplicial chart $\psi_{\alpha}: U_{\alpha} \rightarrow V_{\alpha}$ on $M$ for which $U \cap U_{\alpha}$ is nonempty, the composite $\left(f_{U \cap U_{\alpha}}\right) \circ\left(\left.\psi_{\alpha}\right|_{U \cap U_{\alpha}}\right)^{-1}: \psi_{\alpha}\left(U \cap U_{\alpha}\right) \rightarrow R$ is piecewise smooth (linear).

This definition makes sense since simplicial charts are linearly related.

3.3. Definition. The sheaf $\mathbf{A}(M)$ of piecewise smooth functions on $M$ is the sheaf associated to the presheaf which assigns to each small open subset $U$ of $M$ the algebra $A(U)$ of piecewise smooth functions on $U$ and to each inclusion $U_{1} \subseteq U_{2}$ of small open subsets of $M$ the map $A\left(U_{2}\right) \rightarrow A\left(U_{1}\right)$ given by restriction.

\subsection{LEMMA. The sheaf $\mathrm{A}(M)$ is fine.}

Proof. Let $U$ be an open cover of $M$ by small open subsets. Without loss of generality assume that $U$ is neighborhood finite. Using Dieudonne's "shrinking lemma" there is a neighborhood finite refinement $U^{\prime}$ of $U$ by small open subsets such that for every $U \in U$ there is a $U^{\prime} \in U^{\prime}$ for which the closure of $U^{\prime}$ is contained in $U$. For any $U \in U$ one can (inductively) construct a piecewise smooth function $f: U \rightarrow R^{+}$which is nonzero on $U^{\prime}$ and whose support is the closure of $U^{\prime}$. For each $U_{j} \in U$ let $h_{j}=\Sigma f_{i}$ where the summation is taken over all 
$U_{i} \in U^{\prime}$ for which $U_{i} \subseteq U_{j}$ and where $f_{i}$ is the corresponding piecewise smooth function described above. Then $\left\{\phi_{j}=h_{j} /\left(\Sigma_{k} h_{k}\right)\right\}$ is a piecewise smooth partition of unity subordinate to $U$. Q.E.D.

Again let $U$ be a small open subset of the simplicial space $M$ and let $(U, \Psi)$ be a packet on $U$. Here is an alternate description of the algebra $A(U)$ of piecewise smooth functions defined on $U$ : For each simplicial chart $\left(U_{\alpha}, \psi_{\alpha}\right)$ in $\Psi$ let

$$
A\left(U_{\alpha}\right)=\left\{f: U_{\alpha} \rightarrow R: f \circ \psi_{\alpha}^{-1} \in A\left(\psi_{\alpha}\left(U_{\alpha}\right)\right)\right\}
$$

and let $\left(\psi_{\alpha}^{-1}\right)^{*}: A\left(U_{\alpha}\right) \rightarrow A\left(\psi_{\alpha}\left(U_{\alpha}\right)\right)$ be the isomorphism given by $\left(\psi_{\alpha}^{-1}\right)^{*}(f)=$ $f \circ \psi_{\alpha}^{-1}$. The following lemma is immediate.

3.5. Lemma. There is an isomorphism identifying $A(U)$ as the subalgebra of the direct product $X A\left(U_{\alpha}\right)$, the product taken over all $\alpha$ in the index set $A$ of $\Psi$, consisting of those $\left(f_{\alpha}\right)$ for which

$$
\rho^{*}\left(s_{\alpha \beta}, s_{\alpha}\right)\left(\psi_{\alpha}^{-1}\right)^{*}\left(f_{\alpha}\right)=\left(\left(\left.\psi_{\beta}\right|_{U_{\alpha} \cap U_{\beta}}\right) \circ\left(\left.\psi_{\alpha}\right|_{U_{\alpha} \cap U_{\beta}}\right)^{-1}\right)^{*} \rho^{*}\left(s_{\beta \alpha}, s_{\beta}\right)\left(\psi_{\beta}^{-1}\right)^{*}\left(f_{\beta}\right)
$$

for $\alpha$ and $\beta$ in $A$.

For every simplicial chart $\left(U_{\alpha}, \psi_{\alpha}\right)$ in $\Psi$ let $E\left(U_{\alpha}\right)$ be the free $A\left(U_{\alpha}\right)$-module on generators $d\left(U_{\alpha}\right)\left(f_{\alpha}\right), f_{\alpha} \in A\left(U_{\alpha}\right)$, and let $\left(\psi_{\alpha}^{-1}\right)^{*}: E\left(U_{\alpha}\right) \rightarrow E\left(\psi_{\alpha}\left(U_{\alpha}\right)\right)$ be the isomorphism given by

$$
\left(\psi_{\alpha}^{-1}\right)^{*}\left(f_{\alpha} d\left(U_{\alpha}\right) g_{\alpha}\right)=\left(\left(\psi_{\alpha}^{-1}\right)^{*} f_{\alpha}\right) d\left(s_{\alpha}\right)\left(\left(\psi_{\alpha}^{-1}\right)^{*} g_{\alpha}\right) .
$$

3.6. Definition. The module $E(U)$ of piecewise smooth 1-forms on $U$ is the subgroup of the direct product $X E\left(U_{\alpha}\right)$, the product taken over all $\alpha$ in the index set $A$ of $\Psi$, consisting of those $\left(\theta_{\alpha}\right)$ for which

$$
\begin{aligned}
& \rho^{*}\left(s_{\alpha \beta}, s_{\alpha}\right)\left(\psi_{\alpha}^{-1}\right)^{*}\left(\theta_{\alpha}\right) \\
& \quad=\left(\left(\psi_{\beta} \mid U_{\alpha} \cap U_{\beta}\right) \circ\left(\left.\psi_{\alpha}\right|_{\alpha} \cap U_{\beta}\right)^{-1}\right)^{*} \rho^{*}\left(s_{\beta \alpha}, s_{\beta}\right)\left(\psi_{\beta}^{-1}\right)^{*}\left(\theta_{\beta}\right),
\end{aligned}
$$

for $\alpha$ and $\beta$ in $A$, considered as an $A(U)$-module by: for $f \in A(U)$ and $\theta \in E(U)$, $f \cdot \theta=\left(f_{\alpha}\right) \cdot\left(\theta_{\alpha}\right)=\left(f_{\alpha} \theta_{\alpha}\right)$. Let $d(U): A(U) \rightarrow E(U)$ be the derivation given by

$$
d(U)(f)=\left(d\left(U_{\alpha}\right) f_{\alpha}\right)=\left(\left(\psi_{\alpha}\right)^{*} d\left(s_{\alpha}\right)\left(\psi_{\alpha}^{-1}\right)^{*}\left(f_{\alpha}\right)\right) .
$$

The definition of $d(U): A(U) \rightarrow E(U)$ makes sense since for $\alpha$ and $\beta$ in the index set $A$ of $\Psi$ a simple calculation gives

$$
\begin{aligned}
& \rho^{*}\left(s_{\alpha \beta}, s_{\alpha}\right)\left(\psi_{\alpha}^{-1}\right)^{*}\left(d\left(U_{\alpha}\right) f_{\alpha}\right) \\
& =\left(\left(\left.\psi_{\beta}\right|_{\alpha} \cap U_{\beta}\right) \circ\left(\left.\psi_{\alpha}\right|_{U_{\alpha} \cap U_{\beta}}\right)^{-1}\right)^{*} \rho^{*}\left(s_{\beta \alpha}, s_{\beta}\right)\left(\psi_{\beta}^{-1}\right)^{*}\left(d\left(U_{\beta}\right) f_{\beta}\right) .
\end{aligned}
$$


Furthermore $d(U)$ is a derivation since each $d\left(s_{\alpha}\right)$ is a derivation and $\left(\psi_{\alpha}^{-1}\right)^{*}$ is an isomorphism.

For any $q=1,2, \ldots$ we can define the $A(U)$-modules $\Lambda^{q} E(U)$ of piecewise smooth $q$-forms on $U$ and the derivation $d(U): \Lambda^{q} E(U) \rightarrow \Lambda^{q+1} E(U)$ similarly. Since $d\left(s^{n}\right) \circ d\left(s^{n}\right)=0$ for any $n$-corner $s^{n}, d(U) \circ d(U)=0$, and consequently there is a cochain complex $\left(\Lambda^{*} E(U), d(U)\right)$. Using Remark 1.12 and the fact that $T^{*} d\left(s^{n}\right)=d\left(s^{n}\right) T^{*}$ for an invertible simplicial map $T^{*}$ of small open subsets of $s^{n}$ it follows that the cochain complex $\left(\Lambda^{*} E(U), d(U)\right)$ is independent of the packet structure $\Psi$ chosen on $U$.

3.7. Definition. The cochain complex $\left(\Lambda^{*} \mathrm{E}(M), \mathrm{d}(M)\right)$ of sheaves of piecewise smooth forms on the simplicial space $M$ is the cochain complex of sheaves associated to the cochain complex of presheaves which assigns to each small open subset $U$ of $M$ the cochain complex $\left(\Lambda^{*} E(U), d(U)\right)$ of piecewise smooth forms on $U$ and to each inclusion $U_{1} \subseteq U_{2}$ of small open subsets of $M$ the map from $\left(\Lambda^{*} E\left(U_{2}\right), d\left(U_{2}\right)\right)$ to $\left(\Lambda^{*} E\left(U_{1}\right), d\left(U_{1}\right)\right)$ given by restriction.

Using Lemma 3.4 one can easily verify that the sheaves $\Lambda^{*} \mathrm{E}(M)$ are fine.

3.8. Definition. The de Rham complex $\left(\Lambda^{*} E(M), d(M)\right)$ of the simplicial space $M$ is the complex of global sections of the cochain complex $\left(\Lambda^{*} \mathrm{E}(M), \mathrm{d}(M)\right)$ of sheaves of piecewise smooth forms on $M$.

Here is the first main result of this paper.

3.9. THEOREM (DE RHAM). For every simplicial space $M$ the homology of the de Rham complex $\left(\Lambda^{*} E(M), d(M)\right)$ is isomorphic to the real cohomology of $M$.

Proof. Since the sheaves $\Lambda^{*} \mathrm{E}(M)$ are fine it remains to verify the appropriate Poincare lemma. The standard proof of the de Rham theorem for smooth manifolds (see [2]) uses no properties of smooth function algebras not also shared by piecewise smooth function algebras.

Let us first observe that since the star shaped property is preserved under linear transformations it makes sense to speak of a small open neighborhood of a point $x_{0} \in M$ which is star shaped.

3.10. Lemma (PoIncarÉ). If $U$ is a small star shaped open neighborhood of the point $x_{0}$ in the simplicial space $M$ then the complex $\left(\Lambda^{*} E(U), d(U)\right)$ is exact.

Proof. Suppose that $U$ is the domain of a packet $(U, \Psi)$. If $\theta \in \Lambda^{q} E(U)$ is a piecewise smooth $q$-form on $U$ such that $d(U)(\theta)=0$ then since $d(U)(\theta)=$ $\left(\left(\psi_{\alpha}\right)^{*} d\left(s_{\alpha}\right)\left(\psi_{\alpha}^{-1}\right)^{*}\left(\theta_{\alpha}\right)\right), d\left(s_{\alpha}\right)\left(\psi_{\alpha}^{-1}\right)^{*}\left(\theta_{\alpha}\right)=0$ for each $\alpha$ and the construction of Lemma 2.8 gives piecewise smooth $(q-1)$-forms $\phi_{\alpha} \in \Lambda^{q-1} E\left(U_{\alpha}\right)$ such that $d\left(s_{\alpha}\right)\left(\phi_{\alpha}\right)=\left(\psi_{\alpha}^{-1}\right) *\left(\theta_{\alpha}\right)$. Consider $\phi=\left(\left(\psi_{\alpha}\right) *\left(\phi_{\alpha}\right)\right)$. By Remark 2.9 and Remark 2.10 


$$
\begin{aligned}
\rho^{*}\left(s_{\alpha \beta},\right. & \left.s_{\alpha}\right)\left(\psi_{\alpha}^{-1}\right)^{*}\left(\left(\psi_{\alpha}\right)^{*}\left(\phi_{\alpha}\right)\right) \\
& =\left(\left(\psi_{\beta} \mid U_{\alpha} \cap U_{\beta}\right) \circ\left(\psi_{\alpha} \mid U_{\alpha} \cap U_{\beta}\right)^{-1}\right)^{*} \rho^{*}\left(s_{\beta \alpha}, s_{\beta}\right)\left(\psi_{\beta}^{-1}\right)^{*}\left(\left(\psi_{\beta}\right)^{*}\left(\phi_{\beta}\right)\right)
\end{aligned}
$$

for all $\alpha$ and $\beta$ in the index set $A$ of $\Psi$ so that $\phi \in \Lambda^{q-1} E(U)$. Since $d(U)(\phi)=$ $\theta$, the conclusion follows. Q.E.D.

3.11. REMARK. One can define integration of singular simplicial simplices on simplicial spaces and prove Stokes' theorem for simplicial spaces. Integration thus provides a morphism from the de Rham complex of a simplicial space to its cochain complex of singular simplicial cochains. In this context the de Rham theorem for simplicial spaces states that this map induces an isomorphism in cohomology.

3.12. Remark. Let $\mathbf{M}$ be a smooth manifold. Each piecewise smooth triangulation $T: M \rightarrow M$ of $\mathbf{M}$ by a simplicial space $M$ induces a monomorphism $T^{*}: A(\mathrm{M}) \rightarrow A(M)$ from the standard smooth function algebra $A(\mathrm{M})$ of $\mathrm{M}$ to the piecewise smooth function algebra $A(M)$ of $M$ which extends to a monomorphism of cochain complexes $T^{*}:\left(\Lambda^{*} E(\mathbf{M}), d(\mathbf{M})\right) \rightarrow\left(\Lambda^{*} E(M), d(M)\right)$ where $\left(\Lambda^{*} E(\mathbf{M}), d(\mathbf{M})\right)$ denotes the standard smooth de Rham complex of $\mathbf{M}$ and $\left(\Lambda^{*} E(M), d(M)\right)$ denotes the piecewise smooth de Rham complex of $M$. Furthermore $T^{*}$ induces an isomorphism of de Rham cohomologies.

4. Coordinate systems. In this section local coordinates are defined on each packet $(U, \Psi)$ of a simplicial space $M$. A coordinate system $u=\left\{u_{*}\right\}$ on the packet $(U, \Psi)$ is roughly a collection of simplicial functions $u_{k}: U \rightarrow R$ such that for every simplicial chart $\psi_{\alpha}: U_{\alpha} \rightarrow V_{\alpha}$ of $\Psi,\left.u_{k}\right|_{U_{\alpha}}=0$ except for a subset of $i$ functions $u_{k} \in u$ and the set of functions $u_{k} \circ \psi_{\alpha}^{-1}: V_{\alpha} \rightarrow R$ for $u_{k}$ in this subset forms a "coordinate system" on $V_{\alpha}$.

We first recall that a packet $(U, \Psi)$ on a simplicial space $M$ consists of an open subset $U$ of $M$ and a "maximal" collection $\Psi$ of related simplicial charts $\psi_{\alpha}: U_{\alpha} \rightarrow V_{\alpha} \subseteq s_{\alpha}^{i}, \alpha$ in some finite index set $A$, where $U_{\alpha} \subseteq U$ and $V_{\alpha}$ lies in the subset $s_{\alpha}^{i}$ of the $i$-dimensional vector space $R_{\alpha}^{i}$. Now let $x_{0} \in U$ be a point with respect to which $U$ is a small open neighborhood. For each $\alpha \in A$ we will consider sets $\left\{v_{\alpha}^{*} \in R_{\alpha}^{i}:{ }^{*} \in A_{\alpha}\right\}$ of vectors based at the corresponding points $\psi_{\alpha}\left(x_{0}\right) \in V_{\alpha}, A_{\alpha}$ denoting some finite index set, such that

(1) for every $\alpha \in A,\left\{v_{\alpha}^{*} \in R_{\alpha}^{i}: * \in A_{\alpha}\right\}$ is a basis of $R_{\alpha}^{i}$, and (2) if $\psi_{\alpha}: U_{\alpha} \rightarrow V_{\alpha} \subseteq s_{\alpha}^{i}$ and $\psi_{\beta}: U_{\beta} \rightarrow V_{\beta} \subseteq s_{\beta}^{j}$ are simplicial charts of $\Psi$ for which $U_{\alpha} \subseteq U_{\beta}$ then there is a subset of the set $\left\{v_{\beta}^{*} \in R_{\beta}^{j}: * \in A_{\beta}\right\}$ which is the image of the set $\left\{v_{\alpha}^{*} \in R_{\alpha}^{i}: * \in A_{\alpha}\right\}$ under the unique extension of $\psi_{\beta} \circ \psi_{\alpha}^{-1}$ : $V_{\alpha} \rightarrow V_{\beta}$ to a linear transformation of vector spaces. 
It is not difficult to show that such sets of vectors exist (see [3]).

Since $\left\{v_{\alpha}^{*} \in R_{\alpha}^{i}:{ }^{*} \in A_{\alpha}\right\}$ is a basis of $R_{\alpha}^{i}$ there is a dual basis $\left\{y_{\alpha}^{*}:{ }^{*} \in A_{\alpha}\right\}$ of $R_{\alpha}^{i}$ for every $\alpha \in A$. Conditions (*) imply that if $\left(U_{\alpha}, \psi_{\alpha}\right)$ and $\left(U_{\beta}, \psi_{\beta}\right)$ are simplicial charts of $\Psi$ for which $U_{\alpha} \subseteq U_{\beta}$ and $y_{\beta} \in\left\{y_{\beta}^{*}: * \in A_{\beta}\right\}$ then $y_{\beta} \circ \psi_{\beta}^{\circ}$ $\psi_{\alpha}^{-1}: V_{\alpha} \rightarrow R$ is either an element of $\left\{y_{\alpha}^{*}: * \in A_{\alpha}\right\}$ or identically zero; in fact each $y_{\alpha} \in\left\{y_{\alpha}^{*}: * \in A_{\alpha}\right\}$ is uniquely of the form $y_{\beta} \circ \psi_{\beta} \circ \psi_{\alpha}^{-1}: V_{\alpha} \rightarrow R$ for some $y_{\beta} \in\left\{y_{\beta}^{*}: * \in A_{\beta}\right\}$. For each $\alpha \in A$ and $k \in A_{\alpha}$ let $u_{\alpha}^{k}=y_{\alpha}^{k} \circ \psi_{\alpha}: U_{\alpha} \rightarrow R$.

We define a set $u=\left\{u_{*}\right\}$ of simplicial functions on $U$ as follows: The set $\left\{u_{\alpha}^{*}: * \in A_{\alpha}\right.$ and $\left.\alpha \in A\right\}$ can be partitioned into subsets $u(k)$ in such a way that $u_{\alpha} \in\left\{u_{\alpha}^{*}:{ }^{*} \in A_{\alpha}\right\}$ and $u_{\beta} \in\left\{u_{\beta}^{*}:{ }^{*} \in A_{\beta}\right\}$ are in $u(k)$ iff $\left.u_{\alpha}\right|_{U_{\alpha} \cap U_{\beta}} \in\left\{u_{\gamma}^{*}\right.$ : $\left.* \in A_{\gamma}\right\}$ and $u_{\beta} !_{U_{\alpha} \cap U_{\beta}} \in\left\{u_{\gamma}^{*}: * \in A_{\gamma}\right\}$, where $U_{\gamma}=U_{\alpha} \cap U_{\beta}$, and furthermore $u_{\alpha \mid U_{\alpha} \cap U_{\beta}}=u_{\beta} \mid U_{\alpha} \cap U_{\beta}$. Associate a simplicial function $u_{k}: U \rightarrow R$ to each such set $u(k)$ by letting $u_{k}(x)=u_{\alpha}(x)$ for any $x \in \bigcup\left\{U_{\alpha}: u_{\alpha} \in u(k)\right\}$ and any $u_{\alpha} \in u(k)$, and letting $u_{k}(x)=0$ otherwise. In other words (see Lemma 3.5) $u_{k}=\left(\left(u_{k}\right)_{\alpha}\right)$ where $\left(u_{k}\right)_{\alpha}=u_{\alpha}$ if $u_{\alpha} \in u(k)$ and $\left(u_{k}\right)_{\alpha}=0$ otherwise.

4.1. Definition. A coordinate system on the packet $(U, \Psi)$ is a collection $u=\left\{u_{*}\right\}$ of simplicial functions $u_{k}: U \rightarrow R$ as constructed above. The point $x_{0} \in U$ is the origin of the coordinate system $u$.

4.2. REMARK. If $u$ is a coordinate system on the packet $(U, \Psi), u_{k} \in U$ and $\left(U_{\alpha}, \psi_{\alpha}\right)$ is any simplicial chart of $\Psi$ then by definition either $\left.u_{k}\right|_{U_{\alpha}}=$ $u_{\alpha}^{k} \in\left\{u_{\alpha}^{*}: * \in A\right\}$ or $\left.u_{k}\right|_{U_{\alpha}}=0$.

4.3. REMARK. If $(U, \Psi)$ is a packet, $u$ is a coordinate system on $(U, \Psi)$ and $W$ is a small open subset of $U$ then there are induced coordinate systems on $W$ associated to the packet on $W$ induced by $\Psi$. For suppose that $u$ is described by vectors $\left\{v_{\alpha}^{*} \in R_{\alpha}^{i}: * \in A\right.$ and $\left.\alpha \in A\right\}$ based at the points $\psi_{\alpha}\left(x_{0}\right) \in V_{\alpha}$ for every $\alpha \in A$ where $x_{0} \in U$ is a point with respect to which $U$ is a small open neighborhood. If $x_{1} \in W$ is any point with respect to which $W$ is a small open neighborhood then there is an induced coordinate system on $W$ described by parallel translating the vectors $\left\{v_{\alpha}^{*} \in R_{\alpha}^{i}: * \in A_{\alpha}\right\}$ in $R_{\alpha}^{i}$ to vectors based at the corresponding points $\psi_{\alpha}\left(x_{1}\right)$ for every $\alpha \in A$ for which $W \cap U_{\alpha}$ is nonempty.

4.4. REMARK. If $\left(U, \Psi^{\prime}\right)$ and $\left(U, \Psi^{\prime \prime}\right)$ are related packets on the same small open subset $U$ and $u^{\prime}$ is a coordinate system on $\left(U, \Psi^{\prime}\right)$ then $u^{\prime}$ gives rise to a related coordinate system $u^{\prime \prime}$ on $\left(U, \Psi^{\prime \prime}\right)$ as follows: In Remark 1.12 it was shown that for every simplicial chart $\left(U_{\alpha}, \psi_{\alpha}\right)$ in $\Psi^{\prime}$ there is a simplicial chart $\left(U_{\beta}, \psi_{\beta}\right)$ in $\Psi^{\prime \prime}$ for which $U_{\alpha}=U_{\beta}$. If $\left\{v_{\alpha}^{*} \in R_{\alpha}^{i}: * \in A_{\alpha}\right\}$ is the set of vectors in $R_{\alpha}^{i}$ which gives rise to $u^{\prime}$, let $\left\{v_{\beta}^{*} \in R_{\beta}^{j}\right.$ : $\left.{ }^{*} \in A_{\beta}\right\}$ be the image of $\left\{v_{\alpha}^{*} \in R_{\alpha}^{i}\right.$ : $\left.* \in A_{\alpha}\right\}$ under the unique extension of $\psi_{\beta} \circ \psi_{\alpha}^{-1}: V_{\alpha} \rightarrow V_{\beta}$ to an invertible linear transformation of vector spaces. The set of all such sets of vectors $\left\{v_{\beta}^{*} \in\right.$ $R_{\beta}^{j}: * \in A_{\beta}$ \} gives rise to a coordinate system $u^{\prime \prime}$ on $\left(U, \Psi^{\prime \prime}\right)$. Since each 
coordinate function $y_{\beta} \in\left\{y_{\beta}^{*}: * \in A_{\beta}\right\}$ on a particular $R_{\beta}^{j}$ associated to the basis $\left\{v_{\beta}^{*} \in R_{\beta}^{j}: * \in A_{\beta}\right\}$ of $R_{\beta}^{j}$ can be described with respect to the corresponding coordinate functions $\left\{y_{\alpha}^{*}: * \in A_{\alpha}\right\}$ on $R_{\alpha}^{i}$ by $y_{\beta}=y_{\alpha} \circ \psi_{\alpha} \circ \psi_{\beta}^{-1}$ for some $y_{\alpha} \in$ $\left\{y_{\alpha}^{*}: * \in A_{\alpha}\right\}, u_{\beta}=y_{\beta} \circ \psi_{\beta}=y_{\alpha} \circ \psi_{\alpha}=u_{\alpha}$.

4.5. LEMMA. If $u^{\prime}$ and $u^{\prime \prime}$ are coordinate systems on the packet $(U, \Psi)$. which have the same origin then for every $u_{i} \in u^{\prime}$ there are unique real constants $c_{i j}$ for which $u_{i}=\Sigma_{j} c_{i j} u_{j}$ for $u_{j} \in u^{\prime \prime}$.

Proof. Observe that if $u_{i} \in u^{\prime}$ and $\left(U_{\alpha}, \psi_{\alpha}\right)$ is a simplicial chart of $\Psi$ for which $u_{i \mid U_{\alpha}}=u_{\alpha}^{i} \in\left\{u_{\alpha}^{*}: * \in A_{\alpha}^{\prime}\right\}$ then

$$
\left.u_{i}\right\}_{U_{\alpha}}=u_{\alpha}^{i}=y_{\alpha}^{i} \circ \psi_{\alpha}=\sum_{j} c_{i j}^{\alpha} y_{\alpha}^{i} \circ \psi_{\alpha}=\sum_{i} c_{i j}^{\alpha} u_{\alpha}^{i}=\sum_{j} c_{i j}^{\alpha} u_{j} \mid U_{\alpha}
$$

for unique real constants $c_{i j}^{\alpha}$ and $u_{j} \in u^{\prime \prime}$. Now suppose that $\left(U_{\alpha}, \psi_{\alpha}\right)$ and $\left(U_{\beta}, \psi_{\beta}\right)$ are simplicial charts of $\Psi$ for which $U_{\alpha} \subseteq U_{\beta}$ and that $u_{i} \in u^{\prime}$ is such that $\left.u_{i}\right|_{U_{\alpha}}=u_{\alpha}^{i} \in\left\{u_{\alpha}^{*}: * \in A_{\alpha}^{\prime}\right\}$ (hence $\left.u_{i}\right|_{U_{\beta}}=u_{\beta}^{i} \in\left\{u_{\beta}^{*}: * \in A_{\beta}^{\prime}\right\}$ ). Then since either $u_{j \mid U_{\alpha}}=u_{\alpha}^{j} \in\left\{u_{\alpha}^{*}: * \in A_{\alpha}^{\prime \prime}\right\}$ or $\left.u_{j}\right|_{U_{\alpha}}=0$ for each $u_{j} \in u^{\prime \prime}$,

$$
\sum_{j} c_{i j}^{\alpha} u_{\alpha}^{j}=\left.u_{i}\right|_{\alpha}=\left(\left.u_{i}\right|_{U_{\beta}}\right)_{\mid U_{\alpha}}=\sum_{j} c_{i j}^{\beta} u_{\beta}^{j}
$$

where the summations are taken over all $j$ for which $u_{\alpha}^{j}=\left.u_{j}\right|_{U_{\alpha}} \in\left\{u_{\alpha}^{*}: * \in A_{\alpha}^{\prime \prime}\right\}$. Thus $\Sigma_{j} c_{i j}^{\alpha} y_{\alpha}^{j}=\Sigma_{j} c_{i j}^{\beta} y_{\alpha}^{j}$ which implies that $c_{i j}^{\alpha}=c_{i j}^{\beta}$ for every $j$ for which $u_{j} \in u^{\prime \prime}$ and $\left.u_{j}\right|_{U_{\alpha}}=u_{\alpha}^{j} \in\left\{u_{\alpha}^{*}: * \in A_{\alpha}^{\prime \prime}\right\}$.

For each $u_{i} \in u^{\prime}$ and each $u_{j} \in u^{\prime \prime}$ for which there is a simplicial chart $\left(U_{\alpha}, \psi_{\alpha}\right)$ of $\Psi$ for which $\left.u_{i}\right|_{U_{\alpha}}=u_{\alpha}^{i} \in\left\{u_{\alpha}^{*}: * \in A_{\alpha}^{\prime}\right\}$ and $\left.u_{j}\right|_{U_{\alpha}}=u_{\alpha}^{j} \in\left\{u_{\alpha}^{*}:\right.$ $\left.* \in A^{\prime \prime}\right\}$ let $c_{i j}=c_{i j}^{\alpha}$ for any $\alpha$ for which $\left.u_{i}\right|_{U_{\alpha}}=u_{\alpha}^{i} \in\left\{u_{\alpha}^{*}: * \in A_{\alpha}^{\prime}\right\}$ and $\left.u_{j}\right|_{U_{\alpha}}=u_{\alpha}^{j} \in\left\{u_{\alpha}^{*}: * \in A_{\alpha}^{\prime \prime}\right\}\left(c_{i j}\right.$ is independent of the choice of $\alpha \in A$ as a result of the preceding paragraph). Since either $\left.u_{j}\right|_{U_{\alpha}}=u_{\alpha}^{j} \in\left\{u_{\alpha}^{*}:{ }^{*} \in A_{\alpha}^{\prime \prime}\right\}$ or $\left.u_{j}\right|_{U_{\alpha}}=$ 0 for each $u_{j} \in u^{\prime \prime},\left.\left(\Sigma_{j} c_{i j} u_{j}\right)\right|_{U_{\alpha}}=\Sigma_{j} c_{i j}^{\alpha} u_{\alpha}^{j}=\left.u_{i}\right|_{U_{\alpha}}$ for any simplicial chart $\left(U_{\alpha}, \psi_{\alpha}\right)$ of $\Psi$ so that $u_{i}=\Sigma_{j} c_{i j} u_{j}$. Q.E.D.

Now let $u$ be a coordinate system on the packet $(U, \Psi)$.

4.6. Definition. For each $u_{i} \in u$ the star St $u_{i}$ of $u_{i}$ is the union of the set of all simplicial domains $U_{\alpha}$ of $\Psi$ for which $\left.u_{i}\right|_{U_{\alpha}}=u_{\alpha}^{i} \in\left\{u_{\alpha}^{*}:{ }^{*} \in A_{\alpha}\right\}$.

4.7. Definition. For each $u_{i} \in u$ the derivation $\partial / \partial u_{i}: A(U) \rightarrow A\left(\right.$ St $\left.u_{i}\right)$ is described as follows: For every simplicial chart $\left(U_{\alpha}, \psi_{\alpha}\right)$ of $\Psi$ for which $\left.u_{i}\right|_{\alpha}=u_{\alpha}^{i} \in\left\{u_{\alpha}^{*}: * \in A_{\alpha}\right\}$ let

$$
\left(\left(\partial / \partial u_{i}\right) f\right)_{\alpha}=\psi_{\alpha}^{*}\left(\partial\left(f_{\alpha} \circ \psi_{\alpha}^{-1}\right) / \partial y_{\alpha}^{i}\right)
$$

and for any simplicial chart $\left(U_{\gamma}, \psi_{\gamma}\right)$ of $\Psi$ for which there is a simplicial chart $\left(U_{\alpha}, \psi_{\alpha}\right)$ of $\Psi$ such that $U_{\gamma} \subseteq U_{\alpha}$ and $\left.u_{i}\right|_{U_{\alpha}}=u_{\alpha}^{i} \in\left\{u_{\alpha}^{*}:{ }^{*} \in A_{\alpha}\right\}$ let 


$$
\left(\left(\partial / \partial u_{i}\right) f\right)_{\gamma}=\left(\left.\psi_{\alpha}\right|_{U_{\gamma}}\right)^{*} \rho^{*}\left(s_{\alpha \gamma}, s_{\alpha}\right)\left(\psi_{\alpha}^{-1}\right) *\left(\left(\partial / \partial u_{i}\right) f\right)_{\alpha}
$$

This definition makes sense since taking the restriction to the face $s_{i}$ of a piecewise smooth function defined on $s^{n}$ commutes with taking partial derivatives with respect to coordinate functions describing the face $s_{i}$ of $s^{n}$ so that whenever $\left(U_{\alpha}, \psi_{\alpha}\right)$ and $\left(U_{\beta}, \psi_{\beta}\right)$ are simplicial charts of $\Psi$ for which $y_{\alpha}^{i} \circ \psi_{\alpha \mid U_{\alpha} \cap U_{\beta}}=$ $y_{\beta}^{j} \circ \psi_{\beta \mid U_{\alpha} \cap U_{\beta}}, y_{\alpha}^{i} \in\left\{y_{\alpha}^{*}: * \in A_{\alpha}\right\}$ and $y_{\beta}^{j} \in\left\{y_{\beta}^{*}: * \in A_{\beta}\right\}, \rho^{*}\left(s_{\alpha \beta}, s_{\alpha}\right) \cdot$ $\left(\partial\left(f_{\alpha} \circ \psi_{\alpha}^{-1}\right) / \partial y_{\alpha}^{i}\right)=\left(\left(\psi_{\beta \mid U_{\alpha} \cap U_{\beta}}\right) \circ\left(\psi_{\alpha \mid U_{\alpha} \cap U_{\beta}}\right)^{-1}\right)^{*} \rho^{*}\left(s_{\beta \alpha}, s_{\beta}\right)\left(\partial\left(f_{\beta} \circ \psi_{\beta}^{-1}\right) / \partial y_{\beta}^{j}\right)$.

4.8. Proposition. Let $(U, \Psi)$ be a packet on the simplicial space $M$ and let $u$ be a coordinate system on $(U, \Psi)$. The $A(U)$-module $E(U)$ of piecewise smooth 1-forms on $U$ may be identified as the free Abelian group on generators $f d u_{i}$ where $u_{i} \in u$ and $f \in A\left(\mathrm{St} u_{i}\right)$ with an $A(U)$-module structure given by: for $f \in A(U)$ and $\theta=\Sigma f_{i} d u_{i} \in E(U), f \cdot \theta=\Sigma\left(\left.f\right|_{\mathrm{St} u_{i}}\right) f_{i} d u_{i}$. The derivation $d(U)$ : $A(U) \rightarrow E(U)$ of piecewise smooth functions may be identified as $d(U) f=$ $\Sigma\left(\partial f / \partial u_{i}\right) d u_{i}$ where $\partial f / \partial u_{i} \in A\left(\right.$ St $\left.u_{i}\right)$ for each $i$.

Proof. Let $f d u_{i}$ be any generator as described in the statement of the proposition and $\theta=\left(\theta_{\alpha}\right)$ where $\theta_{\alpha}=\left(\left.f\right|_{U_{\alpha}}\right) d u_{\alpha}^{i} \in E\left(U_{\alpha}\right)$ if $\left.u_{i}\right|_{U_{\alpha}}=u_{\alpha}^{i} \in\left\{u_{\alpha}^{*}\right.$ : $\left.* \in A_{\alpha}\right\}$ and $\theta_{\alpha}=0$ otherwise; this makes sense since whenever $\left.u_{i}\right|_{U_{\alpha}}=u_{\alpha}^{i} \in$ $\left\{u_{\alpha}^{*}: * \in A_{\alpha}\right\}, U_{\alpha} \subseteq$ St $u_{i}$. To show that $\theta$ is actually an element of $E(U)$ we must show that if $\alpha$ and $\beta$ are in the index set $A$ of $\Psi$ such that $U_{\alpha} \subseteq U_{\beta}$ then $\left(\left.\left(\left.f\right|_{U_{\beta}}\right)\right|_{U_{\alpha}}\right) d\left(\left.\left(\left.u_{i}\right|_{U_{\beta}}\right)\right|_{U_{\alpha}}\right)=\left(\left.f\right|_{U_{\alpha}}\right) d\left(\left.u_{i}\right|_{U_{\alpha}}\right)$; however this is obvious.

If, on the other hand, $\theta=\left(\theta_{\alpha}\right)=\left(\Sigma f_{\alpha}^{i} d u_{\alpha}^{i}\right) \in E(U)$ where $\theta_{\alpha}=\Sigma f_{\alpha}^{i} d u_{\alpha}^{i} \in$ $E\left(U_{\alpha}\right)$ then "compatibility" of the forms $\theta_{\alpha}$ means that if $\alpha$ and $\beta$ are in the index set $A$ of $\Psi, U_{\alpha} \subseteq U_{\beta}, \theta_{\alpha}=\Sigma f_{\alpha}^{i} d u_{\alpha}^{i}$ and $\theta_{\beta}=\Sigma f_{\beta}^{j} d u_{\beta}^{j}$ then for each $i$ there is a unique $j$ such that $\left(\left.f_{\beta}^{j}\right|_{U_{\alpha}}\right) d\left(\left.u_{\beta}^{j}\right|_{U_{\alpha}}\right)=f_{\alpha}^{i} d u_{\alpha}^{i}$ (and for each $j$ either $\left.u_{\beta}^{j}\right|_{U_{\alpha}}=0$ or there is a unique $i$ such that $\left.\left(\left.f_{\beta}^{j}\right|_{U_{\alpha}}\right) d\left(\left.u_{\beta}^{j}\right|_{U_{\alpha}}\right)=f_{\alpha}^{i} d u_{\alpha}^{i}\right)$. For each $u_{i} \in u$ one can thus obtain a compatible collection $\left\{f_{\alpha}^{i} \in A\left(U_{\alpha}\right)\right\}$ of piecewise smooth functions defined on the simplicial domains $U_{\alpha}$ of $\Psi$ for which $\left.u_{i}\right|_{U_{\alpha}}=u_{\alpha}^{i} \in\left\{u_{\alpha}^{*}: * \in A_{\alpha}\right\}$. Since the subsets $U_{\alpha}$ form a neighborhood finite closed cover of $U$ we may define a piecewise smooth function $f_{i} \in A\left(\right.$ St $u_{i}$ ) for every $u_{i} \in u$ such that $\left.f_{i}\right|_{U_{\alpha}}=f_{\alpha}^{i}$ whenever $U_{\alpha} \subseteq$ St $u_{i}$. Thus $\theta=\Sigma f_{i} d u_{i}$.

The rest of the proposition is immediate. Q.E.D.

The structure of the $A(U)$-module $E(U)$ of piecewise smooth 1 -forms is now clear: A general element $\theta \in E(U)$ may be formally written $\theta=\Sigma f_{i} d u_{i}, u_{i} \in u$ and $f_{i} \in A\left(\right.$ St $\left.u_{i}\right)$. The forms $\left(\left.1\right|_{\text {St } u_{i}}\right) d u_{i} \in E(U)$, where $\left.1\right|_{\text {St } u_{i}} \in A\left(\right.$ St $\left.u_{i}\right)$ are the piecewise smooth functions which are identically 1 on St $u_{i}$, thus form a set of generators for $E(U)$ as an $A(U)$-module. However there are no unique extensions of the coefficient functions $f_{i} \in A\left(\mathrm{St} u_{i}\right)$ to functions of $A(U)$ and thus $E(U)$ is not, in general, free. 
5. Cosimplicial bundles. In this section we define the category of cosimplicial bundles. This category contains a subcategory of vector bundles over simplicial spaces. In general, however, cosimplicial bundles differ from vector bundles since fiber dimensions in cosimplicial bundles are allowed to vary. The reason for introducing cosimplicial bundles is that in the same way every smooth manifold has a cotangent object in the category of smooth vector bundles, every simplicial space has a cotangent object in the category of cosimplicial bundles; these cotangent objects are cosimplicial bundles and not, in general, vector bundles.

In the sequel all vector spaces are finite dimensional and real.

5.1. Definition. A cosimplicial bundle is a continuous surjection $\xi: E \rightarrow$ $M$ from a topological space $E$ to a simplicial space $M$ such that $\xi^{-1}(x)$ is a vector space for each $x \in M$ and

(1) for every simplicial chart $\left(U_{\alpha}, \psi_{\alpha}\right)$ on $M$ there is a vector space $F_{\alpha}$ (unique up to isomorphism) and a map $\phi_{\alpha}: \xi^{-1}\left(U_{\alpha}\right) \rightarrow U_{\alpha} \times F_{\alpha}$ such that $\left.\phi_{\alpha}\right|_{\xi-1}(x): \xi^{-1}(x) \rightarrow\{x\} \times F_{\alpha}$ is a linear surjection for every $x \in U_{\alpha}$,

(2) if $\left(U_{\alpha}, \psi_{\alpha}\right)$ and $\left(U_{\beta}, \psi_{\beta}\right)$ are simplicial charts on $M$ for which $U_{\alpha} \subseteq U_{\beta}$, then $\left.\phi_{\alpha}\right|_{\xi-1(x)}=\left.l_{\alpha \beta} \circ \phi_{\beta}\right|_{\xi-1(x)}$ for all $x \in U_{\alpha}$ and some fixed linear surjection $l_{\alpha \beta}: F_{\beta} \rightarrow F_{\alpha}$,

(3) if $\left(U_{\alpha}, \psi_{\alpha}\right),\left(U_{\beta}, \psi_{\beta}\right)$ and $\left(U_{\gamma}, \psi_{\gamma}\right)$ are simplicial charts on $M$ for which $U_{\alpha} \subseteq U_{\beta} \subseteq U_{\gamma}$ then $l_{\alpha \gamma}=l_{\alpha \beta} \circ l_{\beta \gamma}$, and

(4) if $\left(U_{\alpha}, \psi_{\alpha}\right)$ and $\left(U_{\beta}, \psi_{\beta}\right)$ are simplicial charts on $M$ for which $U_{\alpha}$ is a relatively open subset of $U_{\beta}$ then $\operatorname{dim} F_{\alpha}=\operatorname{dim} F_{\beta}$.

5.2. EXAMPLE. Let $M$ be the simplicial space associated to the polyhedron $K$ which has three vertices $x_{0}, x_{1}$, and $x_{2}$, and which has two one-simplices $\left(x_{0}, x_{i}\right), i=1,2$. Let $\pi_{1}: M \times R^{2} \rightarrow M$ be the product vector bundle over $M$ whose fiber at each point is the vector space $R^{2}$ equipped with the standard basis vectors $e_{i}, i=1,2$ and where $\pi_{1}$ denotes projection onto the first factor. Let $E$ be the subspace of $M \times R^{2}$ consisting of those $(x, v)$ for which $v$ is a multiple of $e_{i}$ if $x \in \mid\left(x_{0}, x_{i}\right) \wedge\left\{x_{0}\right\}$, and $v \in R^{2}$ if $x=x_{0} \quad\left(\left|\left(x_{0}, x_{i}\right)\right|\right.$ denotes the geometric realization of the one-simplex $\left.\left(x_{0}, x_{i}\right), i=1,2\right)$. If $\xi: E \rightarrow M$ is the map given by $\xi(x, v)=x$ for $(x, v) \in E$ then $\xi$ is a cosimplicial bundle. The structure fibers $F_{\alpha}$ of $\xi$ are as follows: $F_{\alpha}$ is the zero vector space if $U_{\alpha}=\left\{x_{0}\right\}$ and otherwise $F_{\alpha}$ is the vector space on one generator $e_{i}$ whenever $U_{\alpha} \subseteq\left|\left(x_{0}, x_{i}\right)\right|, i=1,2$. The structure maps $\phi_{\alpha}: \xi^{-1}\left(U_{\alpha}\right) \rightarrow U_{\alpha} \times F_{\alpha}$ of $\xi$ are as follows: $\phi_{\alpha}\left(x_{0}, v\right)=$ $\left(x_{0}, 0\right)$ if $U_{\alpha}=\left\{x_{0}\right\}$ and otherwise if $U_{\alpha}$ is the domain of a simplicial chart $\left(U_{\alpha}, \psi_{\alpha}\right)$ on $M$ for which $x_{0} \in U_{\alpha}$ and $U_{\alpha} \subseteq\left|\left(x_{0}, x_{i}\right)\right|, i=1,2$, then $\phi_{\alpha}\left(x_{0}, c_{1} e_{1}+c_{2} e_{2}\right)=\left(x_{0}, c_{i} e_{i}\right)$ and $\phi_{\alpha}\left(x, c e_{i}\right)=\left(x, c e_{i}\right)$ if $x \neq x_{0}$. Notice that $\xi$ is clearly not a vector bundle since $\xi^{-1}\left(x_{0}\right)=R^{2}$ and $\xi^{-1}(x)=R^{1}$ for all other $x \in M$. (See Diagram 3.) 


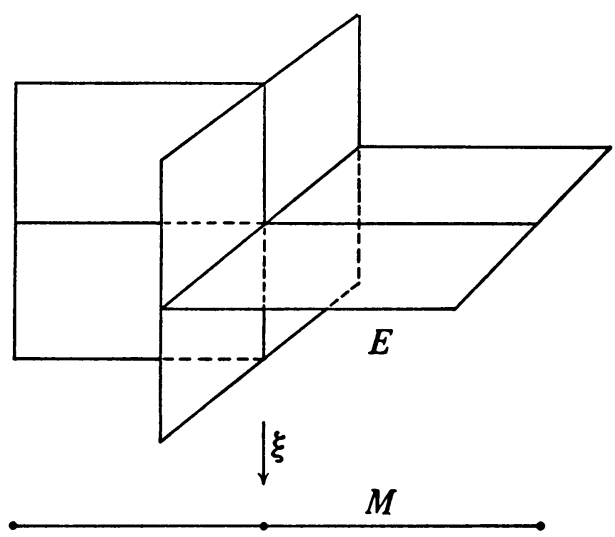

\section{DiAgRAM 3}

For any cosimplicial bundle $\xi: E \rightarrow M$, the inverse image $\xi^{-1}\left(x_{0}\right)$ of any $x_{0} \in M$ is given constructively by the $\left\{F_{\alpha} ; l_{\alpha \beta}\right\}$. This is why in Example 5.2 the structure fiber $R^{0}$ of $\xi$ associated to the simplicial chart with domain $\left\{x_{0}\right\}$ and the inverse image $\xi^{-1}\left(x_{0}\right)=R^{2}$ of $x_{0}$ are so radically different.

5.3. Definition. A morphism $f: \xi_{1} \rightarrow \xi_{2}$ of cosimplicial bundles $\xi_{i}$ : $E_{i} \rightarrow M_{i}, i=1,2$, consists of a simplicial map $f_{1}: M_{1} \rightarrow M_{2}$, a map $f_{2}: E_{1} \rightarrow$ $E_{2}$ taking the fiber $\xi_{1}^{-1}(x)$ over each $x \in M_{1}$ linearly to the fiber $\xi_{2}^{-1}\left(f_{1}(x)\right)$ over $f_{1}(x) \in M_{2}$ and linear maps $f_{\alpha_{2} \alpha_{1}}: F_{\alpha_{1}} \rightarrow F_{\alpha_{2}}$, where the $F_{\alpha_{i}}$ are the structure fibers of $\xi_{i}$ corresponding to simplicial charts $\left(U_{\alpha_{i}}, \psi_{\alpha_{i}}\right)$ on $M_{i}, i=1,2$, for which $f_{1}\left(U_{\alpha_{1}}\right) \subseteq U_{\alpha_{2}}$, such that $\phi_{\alpha_{2}}\left(f_{2}(v)\right)=f_{\alpha_{2} \alpha_{1}}\left(\phi_{\alpha_{1}}(v)\right)$ for $v \in \xi_{1}^{-1}\left(U_{\alpha_{1}}\right)$, and such that if $\left(U_{\alpha_{i}}, \psi_{\alpha_{i}}\right)$ and $\left(U_{\beta_{i}}, \psi_{\beta_{i}}\right)$ are simplicial charts on $M_{i}, i=1,2$, for which $U_{\alpha_{i}} \subseteq U_{\beta_{i}}, f_{1}\left(U_{\alpha_{1}}\right) \subseteq U_{\alpha_{2}}$ and $f_{1}\left(U_{\beta_{1}}\right) \subseteq U_{\beta_{2}}$ then $f_{\alpha_{2} \alpha_{1}} \circ l_{\alpha_{1} \beta_{1}}=$ $l_{\alpha_{2} \beta_{2}} \circ f_{\beta_{2} \beta_{1}}$.

Cosimplicial bundles and morphisms of cosimplicial bundles form a category.

5.4. REMARK. If $\xi: E \rightarrow M$ is a cosimplicial bundle over the simplicial space $M$ for which $\operatorname{dim} F_{\alpha}=\operatorname{dim} \xi^{-1}(x)=m$ for all simplicial charts $\left(U_{\alpha}, \psi_{\alpha}\right)$ on $M$ and $x \in U_{\alpha}$ then $\xi$ is an $m$-dimensional vector bundle over $M$.

Sections of cosimplicial bundles are defined next. First, however, observe that if $\xi: E \rightarrow M$ is the cosimplicial bundle of Example 5.2 and $s: M \rightarrow E$ is any continuous function for which $\xi \circ s=\mathrm{id}_{M}$, then $s\left(x_{0}\right) \in \xi^{-1}\left(x_{0}\right)=R^{2}$ is necessarily zero. In order to avoid such restrictions on sections of cosimplicial bundles, a certain amount of discontinuity will be allowed in the definition of section.

5.5. Definition. A section of the cosimplicial bundle $\xi: E \rightarrow M$ over the simplicial space $M$ is a function (not necessarily a continuous function) s: $M \rightarrow E$ such that $\xi \circ s=\mathrm{id}_{M}$ and

(1) for each simplicial chart $\left(U_{\alpha}, \psi_{\alpha}\right)$ on $M$ and corresponding structure map 
$\phi_{\alpha}: \xi^{-1}\left(U_{\alpha}\right) \rightarrow U_{\alpha} \times F_{\alpha}$ of $\xi$, the coordinate functions of the transformation $\pi_{2} \circ \phi_{\alpha} \circ s: U_{\alpha} \rightarrow F_{\alpha}$ are piecewise smooth, $\pi_{2}: U_{\alpha} \times F_{\alpha} \rightarrow F_{\alpha}$ denoting projection onto the second factor, and

(2) if $\left(U_{\alpha}, \psi_{\alpha}\right)$ and $\left(U_{\beta}, \psi_{\beta}\right)$ are simplicial charts on $M$ for which $U_{\alpha} \subseteq U_{\beta}$ and $\phi_{\alpha}: \xi^{-1}\left(U_{\alpha}\right) \rightarrow U_{\alpha} \times F_{\alpha}$ and $\phi_{\beta}: \xi^{-1}\left(U_{\beta}\right) \rightarrow U_{\beta} \times F_{\beta}$ are the corresponding structure maps of $\xi$, then $\phi_{\alpha} \circ s=\left.\left(\right.$ id $\left.\times l_{\alpha \beta}\right) \circ\left(\phi_{\beta} \circ s\right)\right|_{U_{\alpha}}$.

The definition of section is well defined since if $\left(U_{\alpha}, \psi_{\alpha}\right)$ and $\left(U_{\beta}, \psi_{\beta}\right)$ are simplicial charts on $M$ for which $U_{\alpha} \subseteq U_{\beta}$ then the map $l_{\alpha \beta}: F_{\beta} \rightarrow F_{\alpha}$ for which $\phi_{\left.\alpha\right|_{\xi-1}(x)}=\left.l_{\alpha \beta} \circ \phi_{\beta}\right|_{\xi-1(x)}$ is a linear transformation.

5.6. REMARK. If $\xi$ is a cosimplicial bundle which is a vector bundle in the sense of Remark 5.4 then the sections of the cosimplicial bundle $\xi$ are precisely the (continuous) piecewise smooth sections of the vector bundle $\xi$.

5.7. REMARK. The set $\Gamma(M, \xi)$ of global sections of the cosimplicial bundle $\xi$ forms an $A(M)$-module with respect to pointwise addition and pointwise multiplication by elements of $A(M)$.

We now construct cotangent objects for simplicial spaces in the category of cosimplicial bundles. The cosimplicial bundle $\xi: E \rightarrow M$ of Example 5.2 is a prototype for this construction: $\xi$ is the cotangent object of $M$. Usage of the term "cotangent object" is justified by Theorem 5.12.

5.8. Construction. For every simplicial space $M$ there is a canonical cosimplicial bundle $\tau(M): T M \rightarrow M$ over $M$ called the cotangent object of $M$ which is constructed as follows.

Let $(U, \Psi)$ be a packet on $M$ and $u$ a coordinate system on $(U, \Psi)$. Let $V$ be the free $R$-module on generators $d u_{k}$ for $u_{k} \in u$, and let $\pi_{1}: U \times V \rightarrow U$ be the product vector bundle where $\pi_{1}$ denotes projection onto the first factor. Let $\pi^{-1}(U)$ be the subspace of $U \times V$ consisting of those ordered pairs $(x, v)$ such that $v=\Sigma c_{i} d u_{i}$ where for each $i$ there is a simplicial chart $\left(U_{\alpha}, \psi_{\alpha}\right)$ of $\Psi$ such that $x \in U_{\alpha}$ and for which $\left.u_{i}\right|_{U_{\alpha}}=u_{\alpha}^{i} \in\left\{u_{\alpha}^{*}: * \in A_{\alpha}\right\}$. Finally let $\pi: \pi^{-1}(U)$ $\rightarrow U$ denote projection onto the first factor.

5.9. Lemma. The map $\pi: \pi^{-1}(U) \rightarrow U$ is a cosimplicial bundle.

Proof. If $x \in U, \pi^{-1}(x)$ is the free $R$-module on generators $d u_{i}$ where for each $u_{i}$ there is a simplicial chart $\left(U_{\alpha}, \psi_{\alpha}\right)$ of $\Psi$ such that $x \in U_{\alpha}$ and for which $\left.u_{i}\right|_{U_{\alpha}}=u_{\alpha}^{i} \in\left\{u_{\alpha}^{*}: * \in A_{\alpha}\right\}$. For each simplicial chart $\left(U_{\alpha}, \psi_{\alpha}\right)$ of $\Psi$ let $F_{\alpha}$ be the free $R$-module on generators $d u_{\alpha}^{l}, u_{\alpha}^{l} \in\left\{u_{\alpha}^{*}: * \in A_{\alpha}\right\}$ and let $\phi_{\alpha}: \pi^{-1}\left(U_{\alpha}\right)$ $\rightarrow U_{\alpha} \times F_{\alpha}$ be the map given by $\phi_{\alpha}\left(x, \Sigma c_{i} d u_{i}\right)=\left(x, \Sigma^{\prime} c_{i} d u_{\alpha}^{i}\right)$ where the summation $\Sigma^{\prime} c_{i} d u_{\alpha}^{i}$ is taken over all $i$ for which $u_{i \mid U_{\alpha}}=u_{\alpha}^{i} \in\left\{u_{\alpha}^{*}: * \in A_{\alpha}\right\}$. If $\left(U_{\alpha}, \psi_{\alpha}\right)$ and $\left(U_{\beta}, \psi_{\beta}\right)$ are simplicial charts of $\Psi$ for which $U_{\alpha} \subseteq U_{\beta}$, let $l_{\alpha \beta}: F_{\beta}$ $\rightarrow F_{\alpha}$ be the map given by $l_{\alpha \beta}\left(\Sigma c_{i} d u_{\beta}^{i}\right)=\Sigma^{\prime} c_{i} d u_{\alpha}^{i}$ where the summation $\Sigma^{\prime} c_{i} d u_{\alpha}^{i}$ is taken over all $i$ for which $\left.u_{\beta}^{i}\right|_{U_{\alpha}}=u_{\alpha}^{i} \in\left\{u_{\alpha}^{*}: * \in A_{\alpha}\right\}$. With this 
structure $\pi$ is clearly a cosimplicial bundle. Q.E.D.

Define the total space $T M$ of $\tau(M)$ by covering $M$ with all possible combinations of packets $(U, \Psi)$ equipped with coordinate systems $u$, and letting $T M=$ $\amalg \pi^{-1}(U) / T$ where $\amalg \pi^{-1}(U)$ is the free union of all the topological spaces $\pi^{-1}(U)$ as constructed above and $T$ is the equivalence relation given as follows: Let $\left(x^{\prime}, v^{\prime}\right) \in \pi^{-1}\left(U^{\prime}\right)$ and $\left(x^{\prime \prime}, v^{\prime \prime}\right) \in \pi^{-1}\left(U^{\prime \prime}\right)$ where $\left(U^{\prime}, \Psi^{\prime}\right)$ and $\left(U^{\prime \prime}, \Psi^{\prime \prime}\right)$ are packets on $M$ equipped with coordinate systems $u^{\prime}$ and $u^{\prime \prime}$, respectively. If $U^{\prime}$ and $U^{\prime \prime}$ are small open neighborhoods of the point $x_{0} \in M$ then $U=U^{\prime} \cap U^{\prime \prime}$ is a small open neighborhood of $x_{0}$. Observe that in this case $\Psi^{\prime}$ and $\Psi^{\prime \prime}$ induce packet structures on $U=U^{\prime} \cap U^{\prime \prime}$ (see Remark 1.15) which are essentially the same (see Remark 1.12), and that $u^{\prime}$ and $u^{\prime \prime}$ induce coordinate systems on $U$, again denoted by $u^{\prime}$ and $u^{\prime \prime}$, respectively, both with origin $x_{0} \in U$ (see Remark 4.3). We define $\left(x^{\prime}, v^{\prime}\right) T\left(x^{\prime \prime}, v^{\prime \prime}\right)$ iff $x^{\prime}=x^{\prime \prime}$ and $v^{\prime \prime}=\Sigma_{i, j} c_{i} c_{i j} d u_{j}$ for $u_{j} \in u^{\prime \prime}$ where $v^{\prime}=\Sigma_{i} c_{i} d u_{i}$, $u_{i} \in u^{\prime}$, and for every $u_{i} \in u^{\prime}, u_{i}=\Sigma_{j} c_{i j} u_{j}$ where $u_{j} \in u^{\prime \prime}$ and $c_{i j} \in R$ ( see Lemma 4.5). Let $\tau(M): T M \rightarrow M$ be the induced projection.

\subsection{Lemma. The map $\tau(M): T M \rightarrow M$ is a cosimplicial bundle.}

Proof. Let $\left(U_{\alpha}, \psi_{\alpha}\right)$ be a simplicial chart on $M$ and let $(U, \Psi)$ be a packet on $M$ for which $U_{\alpha} \subseteq U$. (For example if $x_{0}$ is a point in the face of $U_{\alpha}$ of smallest dimension, we may take $U$ to be the largest small open neighborhood of $x_{0}$ in $M$.) Without loss of generality assume that $\left(U_{\alpha}, \psi_{\alpha}\right)$ is a simplicial chart of $\Psi$. Let $u$ be a coordinate system on $(U, \Psi)$, let $F_{\alpha}$ be the vector space on generators $d u_{\alpha}^{i}$ where each $u_{\alpha}^{i}$ corresponds to a $u_{i} \in u$ for which $\left.u_{i}\right|_{U_{\alpha}}=u_{\alpha}^{i} \in\left\{u_{\alpha}^{*}\right.$ : $* \in A\}$, and define the map $\phi_{\alpha}: \pi^{-1}\left(U_{\alpha}\right) \rightarrow U_{\alpha} \times F_{\alpha}$ by $\phi_{\alpha}\left(x, \Sigma c_{i} d u_{i}\right)=$ $\left(x, \Sigma^{\prime} c_{i} d u_{\alpha}^{i}\right)$ where the summation $\Sigma^{\prime} c_{i} d u_{\alpha}^{i}$ is taken over all $i$ for which $u_{i \mid U_{\alpha}}=$ $u_{\alpha}^{i} \in\left\{u_{\alpha}^{*}: * \in A_{\alpha}\right\}$. To verify that $\tau(M)$ is a cosimplicial bundle it suffices to observe that if $u^{\prime}$ and $u^{\prime \prime}$ are two different coordinate systems on $(U, \Psi)$ and $\phi_{\alpha}^{\prime}: \pi^{-1}\left(U_{\alpha}\right) \rightarrow U_{\alpha} \times F_{\alpha}^{\prime}$ and $\phi_{\alpha}^{\prime \prime}: \pi^{-1}\left(U_{\alpha}\right) \rightarrow U_{\alpha} \times F_{\alpha}^{\prime \prime}$ are the associated structure maps of $\tau(M)$ as described above, then the linear map $l: F_{\alpha}^{\prime} \rightarrow F_{\alpha}^{\prime \prime}$ for which $l \circ \pi_{2} \circ \phi_{\alpha}^{\prime}=\pi_{2} \circ \phi_{\alpha}^{\prime \prime}$ is given by taking each generator $d u_{\alpha}^{i} \in F_{\alpha}^{\prime}$ to $\Sigma_{j} c_{i j} d u_{\alpha}^{j} \in F_{\alpha}^{\prime \prime}$ (see Lemma 4.5). The rest of the proof is immediate. Q.E.D.

5.11. Definition. The cosimplicial bundle $\tau(M): T M \rightarrow M$ constructed above is the cotangent object of $M$.

Here is the second main result of this paper.

5.12. THEOREM. For every simplicial space $M$ there is an $A(M)$-module isomorphism between the module $\Gamma(M, \tau(M))$ of global sections of the cotangent object $\tau(M)$ of $M$ and the module $E(M)$ of global piecewise smooth 1-forms on $M$.

Proof. It suffices to work locally, so let $(U, \Psi)$ be a packet on $M$ and $u$ a coordinate system on $(U, \Psi)$. Each section $s \in \Gamma(U, \tau(M))$ may be written 
$s(x)=\left(x, \Sigma f_{i}(x) d u_{i}\right)$ where each $f_{i}$ is a function defined on St $u_{i}$. From the definition of section it follows that each $f_{i} \in A\left(\mathrm{St} u_{i}\right)$. We define the isomorphism $\Gamma(U, \tau(M)) \rightarrow E(U)$ by taking each such section $s \in \Gamma(U, \tau(M))$ to the piecewise smooth 1 -form $\theta=\Sigma f_{i} d u_{i} \in E(U)$. Q.E.D.

6. Closing remarks. The reference for this section is [3].

A simplicial bundle is a continuous surjection $\xi: E \rightarrow M$ from a topological space $E$ to a simplicial space $M$ which satisfies criteria dual to the defining criteria for cosimplicial bundles. To every simplicial space $M$ a simplicial bundle $\tau^{*}(M)$ over $M$ is associated; $\tau^{*}(M)$ is a tangent object for $M$ not only since $\tau^{*}(M)$ is the dual of the cotangent object $\tau(M)$ of $M$ but also since there is a correspondence between piecewise smooth flows on $M$ and sections of $\tau^{*}(M)$.

Using a relative de Rham theorem for simplicial spaces a de Rham cohomology class $\left[O_{2 n}(\xi, s)\right]$ may be associated to each oriented simplicial bundle $\xi$ over a polyhedron $M$ and each nonzero section $s$ of $\xi$ defined on the $(2 n-1)$-skeleton of $M$. The class $\left[O_{2 n}(\xi, s)\right]$ is an obstruction class since $s$ extends to a nonzero section of $\xi$ defined on the $2 n$-skeleton of $M$ iff $\left[O_{2 n}(\xi, s)\right]=0$. (In fact $\left[O_{2 n}(\xi, s)\right]$ plays exactly the same role for simplicial bundles that the Euler class plays for vector bundles.)

Finally there is a Gauss-Bonnet theorem for combinatorial manifolds. This theorem states if $M$ is a closed oriented combinatorial manifold of dimension $2 n$ then the tangent object $\tau^{*}(M)$ of $M$ is oriented; if $F$ is any piecewise smooth flow on $M$ which has only a finite number of nondegenerate fixed points each of which is in the interior of a $2 n$-simplex of $M$ then $F$ gives rise to a section $X_{F}$ of $\tau^{*}(M)$ which is nonzero on the $(2 n-1)$-skeleton of $M$ and the integral of $O_{2 n}\left(\tau^{*}(M), X_{F}\right)$ is the Euler characteristic of $M$. The proof of this theorem is modeled after Chern's intrinsic proof. The existence of piecewise smooth flows as described in the statement of the Gauss-Bonnet theorem for combinatorial manifolds is easily verified. As a consequence of this verification it also follows that if $M$ is a compact combinatorial manifold whose Euler characteristic is zero then there is a nonvanishing piecewise smooth flow on $M$.

\section{REFERENCES}

1. H. Flanders, Differential forms with applications to the physical sciences, Math. in Sci. and Engineering, vol. 11, Academic Press, New York and London, 1963. MR 28 \#5397.

2. F. Hirzebruch, Neue topologische Methoden in der algebraischen Geometrie, Ergebnisse der Mathematik und ihrer Grenzgebiete, Heft 9, Springer-Verlag, Berlin, 1956; English transl., Die Grundlehren der math. Wissenschaften, Band 131, Springer-Verlag, New York, 1966. MR 18,509; 34 \#2573.

3. M. Penna, Differential geometry on simplicial manifolds, Dissertation, University of Illinois, Urbana-Champaign, Ill., 1974.

DEPARTMENT OF MATHEMATICS, INDIANA-PURDUE UNIVERSITY, INDIANAPOLIS, INDIANA 46205 